\title{
On a multiscale approach to the transient Stokes problem: Dynamic subscales and anisotropic space-time discretization
}

\author{
Santiago Badia *,1, Ramon Codina \\ International Center for Numerical Methods in Engineering (CIMNE), Universitat Politècnica de Catalunya, Jordi Girona 1-3, Edifici C1, 08034 Barcelona, Spain
}

\section{A R T I C L E I N F O}

\section{Keywords:}

Stabilized finite elements

Stokes

Multiscale

Dynamic subscales

Stability

Convergence

\begin{abstract}
A B S T R A C T
In this article, we analyze some residual-based stabilization techniques for the transient Stokes problem when considering anisotropic time-space discretizations. We define an anisotropic time-space discretization as a family of time-space partitions that does not satisfy the condition $h^{2} \leqslant C \delta t$ with $C$ uniform with respect to $h$ and $\delta$ t. Standard residual-based stabilization techniques are motivated by a multiscale approach, approximating the effect of the subscales onto the large scales. One of the approximations is to consider the subscales quasi-static (neglecting their time derivative). It is well known that these techniques are unstable for anisotropic time-space discretizations. We show that the use of dynamic subscales (where the subscales time derivatives are not neglected) solves the problem, and prove optimal convergence and stability results that are valid for anisotropic time-space discretizations. Also the improvements related to the use of orthogonal subscales are addressed.
\end{abstract}

(c) 2008 Elsevier Inc. All rights reserved.

\section{Introduction}

The Stokes system is a well-posed mixed problem by virtue of the so-called inf-sup condition, which ensures pressure stability. At the fully discrete level, the main question is: does the velocity-pressure pair of finite-dimensional spaces satisfy the discrete inf-sup condition? In general, as it is widely known, the answer is not. Unfortunately, condition (12) below does not hold for simple cases, such as equal order velocity-pressure interpolation. Stable pairs, that is to say, pairs satisfying the discrete inf-sup condition (12) are called div-stable in the terminology of Boland and Nicolaides [6]. These pairs must be composed of different interpolation spaces for the velocity and pressure. The design of div-stable elements satisfying the discrete inf-sup condition has motivated a large amount of research (see [9]).

At the continuous level, the steady Stokes problem is well-posed due to the celebrated LBB inf-sup condition, that provides optimal pressure stability for the steady-state problem (see $[23,8,1]$ ). The well-posedness of the transient Stokes problem is more delicate; we refer to [5] for a discussion about this topic.

At the discrete level, an alternative approach to the satisfaction of the discrete inf-sup condition relies on the perturbation (stabilization) of the formulation in a consistent way. This approach is particularly appealing because allows to use the same finite element spaces for velocity and pressure interpolation. This idea was initially suggested in [21].

An abstract framework for the motivation and design of stabilization methods based on a multiscale decomposition of the solution was proposed in $[19,20]$. The idea is to split the continuous solution of our problem into coarse (or finite element)

\footnotetext{
* Corresponding author. Present address: Applied Mathematics and Applications, MS-1320, Sandia National Laboratories, Albuquerque, NM 87185-1320, USA.

E-mail addresses: sbadia@cimne.upc.edu (S. Badia), ramon.codina@upc.edu (R. Codina).

${ }^{1}$ The first author's research was supported by the European Community through the Marie Curie contract NanoSim (MOIF-CT-2006-039522).
} 
and fine (or subgrid) scales. In order to get a feasible numerical method, a cheap approximation of the subgrid scales equations is required. This is the frame we consider herein.

Residual-based stabilized methods were initially motivated for the steady-state case. The extension to the transient problem has been at least involved. Initially, space-time finite element approximations were used (see [25]). Usually, the time derivative of the subgrid component at the subgrid equation is approximated by a source-like term, in order to justify a dependence of the subgrid component with the time step size $\delta t$. This approach, that we call quasi-static subscales, is not consistent because it implies a steady-state solution (in case of being reached) time step dependent. In any case, this approach is unstable for anisotropic time-space discretizations, as pointed out in [4]. We define as anisotropic time-space discretization a family of time-space partitions such that $\delta t$ and the characteristic mesh size $h$ do not satisfy

$$
h^{2} \leqslant C \delta t
$$

for a constant $C$ uniform with respect to $h$ and $\delta$. Roughly speaking, residual-based methods become unstable when the time step size $\delta t$ is reduced without remeshing. In fact, all the existing numerical analyses of residual-based stabilization methods need to assume condition (1).

In the present work, we analyze a new approach in which the subgrid component of the solution is tracked in time and the subgrid time derivative taken into consideration. This new method has been called dynamic subscales, in comparison to the classical quasi-static subscales, where this time derivative is neglected. The motivation of the method and implementation aspects can be found in [15]. In this reference, there is a first stability analysis that shows that this approach is stable, regardless of the time step size. The pressure stability was proved in a very weak dual norm (see Theorem 2 therein). However, there was an open question: are we able to get stronger pressure stability (not in dual norms)? In particular, can we prove the same pressure stability for the transient problem (using dynamic subscales) as we get for the steady-state problem? The proof of these results is much more involved, but we have been able to answer this question affirmatively in this work.

The main contributions of this article are:

- To prove stronger pressure stability for the transient Stokes problem stabilized with dynamic subscales, for time-space not relying on (1). We want to show that we get the same control over the pressure that we have for the steady case.

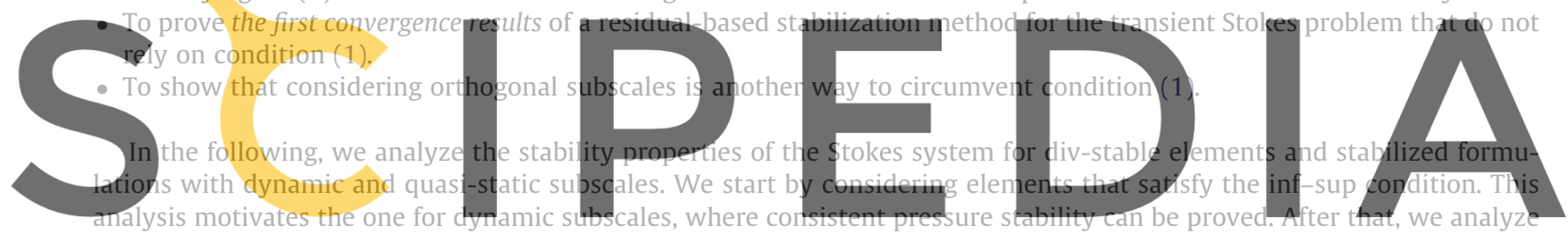
quasi-static subscales in order to show how neglecting the time evolution of the subscale deteriorates the stability properties

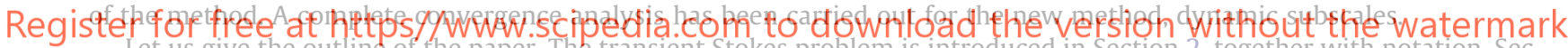
Let us give the outline of the paper. The transient Stokes problem is introduced in Section 2, together with notation. Sec-
tion 3 is devoted to the multiscale stabilization of this mixed problem. Section 4 contains the stability analysis of the Stokes problem for the numerical schemes listed above. Enor estimates have been proved in Section 5 . ve end by summarizing the main results of the article and drawing some conclusions in Section 6.

\section{The Stokes problem}

\subsection{The continuous level}

The Stokes system over a domain $\Omega$ of $\mathbb{R}^{d}(d=2$ or 3 being the space dimension) consists of finding a velocity $\boldsymbol{u}$ and a pressure $p$ such that:

$$
\begin{aligned}
& -v \Delta \boldsymbol{u}+\nabla p=\boldsymbol{f} \text { in } \Omega, \\
& \nabla \cdot \boldsymbol{u}=0 \quad \text { in } \Omega,
\end{aligned}
$$

where $\boldsymbol{f}$ is the force vector and $v$ is the kinematic viscosity. The equation set (2) has to be supplemented with appropriate boundary conditions in order to have a well-posed system. The boundary $\Gamma \equiv \partial \Omega$ is a $(d-1)$-dimensional manifold assumed locally Lipschitz (i.e., smooth enough). For the sake of simplicity we have adopted the homogeneous boundary condition:

$$
\boldsymbol{u}=\mathbf{0} \text { on } \Gamma
$$

for the following stability and convergence analyses.

In order to obtain the weak form of problem (2) we need to introduce some notation. We denote by $L^{p}(\Omega), 1 \leqslant p<\infty$, the space of real functions defined on $\Omega$ with the $p$ th power absolutely integrable with respect to the Lebesgue measure. The space $L^{\infty}(\Omega)$ consists of essentially bounded functions in $\Omega$. The case $p=2$ is of special interest; $L^{2}(\Omega)$ is a Hilbert space endowed with the scalar product $(u, v)$ and its induced norm $\|u\|_{0}$. The Hilbert space $H^{m}(\Omega)$ is the space of functions in $L^{2}(\Omega)$ whose weak derivatives of order less than or equal to $m$ belong to $L^{2}(\Omega), m$ being an integer and $1 \leqslant p \leqslant \infty$. This space is 
endowed with a scalar product and its associated norm $\|\cdot\|_{m}$. Furthermore, we denote by $H_{0}^{1}(\Omega)$ the space of functions of $H^{1}(\Omega)$ vanishing on $\Gamma$ and by $H^{-1}(\Omega)$ its dual space. In general, duality pairings will be denoted with the symbol $\langle\cdot, \cdot\rangle$. Given a function $u \in H_{0}^{1}(\Omega)$ the Poincaré inequality:

$$
\|u\|_{1}^{2} \leqslant C_{\Omega}\|\nabla u\|_{0}^{2}
$$

holds for $\Omega$ with Lipschitz continuous boundary. Therefore, the seminorm $|u|_{1}=\|\nabla u\|_{0}$ in $H^{1}(\Omega)$ is a norm in $H_{0}^{1}(\Omega)$.

We shall often consider $d$-dimensional vector functions with components in one of these spaces. We shall indicate them by boldface letters, for instance $\boldsymbol{H}^{m}(\Omega)=\left(H^{m}(\Omega)\right)^{d}$. In the following, we will not distinguish between scalar products or norms for scalar or vector-valued functions.

Let $\mathscr{V} \equiv \boldsymbol{H}^{1}(\Omega), \mathscr{V}_{0} \equiv \boldsymbol{H}_{0}^{1}(\Omega)$ and $\mathscr{Q} \equiv \boldsymbol{L}^{2}(\Omega) / \mathbb{R}$ denote the real Hilbert spaces for velocity and pressure, respectively, with associated norms $\|\boldsymbol{v}\|_{\mathscr{r}}$ and $\|q\|_{2}$, and let $\boldsymbol{f} \in \mathscr{V}^{\prime}$. Let us define the form related to the viscous term,

$$
a(\boldsymbol{u}, \boldsymbol{v}):=v(\nabla \boldsymbol{u}, \nabla \boldsymbol{v}) \quad \forall \boldsymbol{u}, \boldsymbol{v} \in \mathscr{V} .
$$

This is a bilinear continuous form on $\boldsymbol{H}_{0}^{1}(\Omega)$ which is coercive with respect to $\|\cdot\|_{1}$. The next form is used for the pressure gradient and the incompressibility constraint,

$$
b(\boldsymbol{v}, q):=-(q, \nabla \cdot \boldsymbol{v}) \quad \forall \boldsymbol{v} \in \mathscr{V}_{0}, \forall q \in \mathscr{Q},
$$

which is also continuous with respect to the norms $\|\boldsymbol{q}\|_{0}$ and $\|\boldsymbol{v}\|_{1}$.

Then, the weak form of (2) consist of finding $u \in \mathscr{V}_{0}$ and $p \in \mathcal{Q}$ such that:

$$
\begin{aligned}
& a(\boldsymbol{u}, \boldsymbol{v})+b(\boldsymbol{v}, p)=\langle\boldsymbol{f}, \boldsymbol{v}\rangle \quad \forall \boldsymbol{v} \in \mathscr{V}_{0}, \\
& b(\boldsymbol{u}, q)=0 \quad \forall q \in 2 .
\end{aligned}
$$

The requirement in order for (7) to be well posed reads as follows: there exists a constant $\tilde{\beta}$ such that:

$$
\inf _{q \in 9} \sup _{v \in \mathcal{X}_{0}} \frac{b(v, q)}{\|v\|_{\boldsymbol{\gamma}}\|q\|_{2}} \geqslant \tilde{\beta}>0
$$

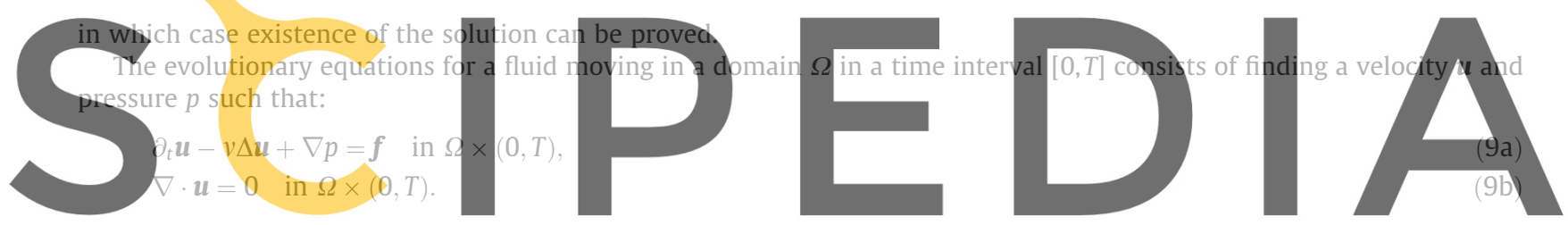

The equation set (9) has to be supplemented with appropriate boundary and initial conditions in order to have a well-posed

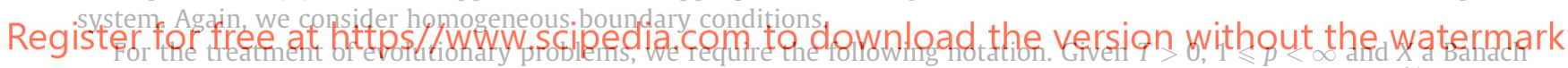

space with norm $\|\cdot\|_{X}$, let $L^{p}(0, T ; X)$ be the space of functions $f:(0, T) \rightarrow X$ such that $\|f\|_{L^{p}(0, T ; X)}=\left(\int_{0}^{T}\|f(s)\|_{X}^{p} d s\right)^{1 / p}<\infty$. In

the case of $\hat{p}=\infty$, vie demand the property sup $\operatorname{sis}_{0 \leqslant T}\|f(s)\|_{X} \leqslant \infty$.

The spaces $\left(\mathscr{V}_{0}\right)_{t} \equiv L^{2}\left(0, T ; \boldsymbol{H}_{0}^{1}(\Omega)\right)$ and $\mathscr{Q}_{t} \equiv \mathscr{D}^{\prime}\left(0, T ; \boldsymbol{L}^{2}(\Omega) / \mathbb{R}\right)$ denote the spaces for velocity and pressure, respectively $\left(\mathscr{D}^{\prime}\right.$ is the space of distributions). Then, the weak form of (9) consists of finding $\boldsymbol{u} \in\left(\mathscr{V}_{0}\right)_{t}$ and $p \in \mathscr{Q}_{t}$ such that:

$$
\begin{aligned}
& \left(\partial_{t} \boldsymbol{u}, \boldsymbol{v}\right)+a(\boldsymbol{u}, \boldsymbol{v})+b(\boldsymbol{v}, p)=\langle\boldsymbol{f}, \boldsymbol{v}\rangle \quad \forall \boldsymbol{v} \in \mathscr{V}_{0} \\
& b(\boldsymbol{u}, q)=0 \quad \forall q \in \mathscr{Q}
\end{aligned}
$$

\subsection{The discrete approach}

We now consider the approximation of the weak form (7) using the finite element approximation theory. Let $\mathscr{V}_{h, 0}$ and $\mathscr{Q}_{h}$ be finite-dimensional subspaces approximating $\mathscr{V}_{0}$ and $\mathscr{2}$, respectively, where the index $h$ refers to the mesh size. The discrete version of system (7) consists of finding $\boldsymbol{u}_{h} \in \mathscr{V}_{h, 0}$ and $p_{h} \in \mathscr{Q}_{h}$ such that:

$$
\begin{aligned}
& a\left(\boldsymbol{u}_{h}, \boldsymbol{v}_{h}\right)+b\left(\boldsymbol{v}_{h}, p_{h}\right)=\left\langle\boldsymbol{f}, \boldsymbol{v}_{h}\right\rangle \quad \forall \boldsymbol{v} \in \mathscr{V}_{h, 0}, \\
& b\left(\boldsymbol{u}_{h}, q_{h}\right)=0 \quad \forall q \in \mathscr{Q}_{h} .
\end{aligned}
$$

The equivalent version of the inf-sup condition at the discrete level holds if there exists a constant $\beta$, independent of the mesh size $h$, such that:

$$
\inf _{q_{h} \in 2_{h}} \sup _{v_{h} \in \mathscr{V}_{h, 0}} \frac{b\left(\boldsymbol{v}_{h}, q_{h}\right)}{\left\|\boldsymbol{v}_{h}\right\|_{\mathscr{V}}\left\|q_{h}\right\|_{\mathscr{Q}}} \geqslant \beta>0,
$$

in which case stability can be proved. 
The space discretization of the evolutionary system (10) consists of finding $\boldsymbol{u}_{h} \in\left(\mathscr{V}_{h, 0}\right)_{t}$ and $p_{h} \in\left(\mathscr{Q}_{h}\right)_{t}$ such that:

$$
\begin{aligned}
& \left(\partial_{t} \boldsymbol{u}_{h}, \boldsymbol{v}_{h}\right)+a\left(\boldsymbol{u}_{h}, \boldsymbol{v}_{h}\right)+b\left(\boldsymbol{v}_{h}, p_{h}\right)=\left\langle\boldsymbol{f}, \boldsymbol{v}_{h}\right\rangle \quad \forall \boldsymbol{v}_{h} \in \mathscr{V}_{h, 0}, \\
& b\left(\boldsymbol{u}_{h}, q_{h}\right)=0 \quad \forall q_{h} \in \mathscr{Q}_{h},
\end{aligned}
$$

being $\left(\mathscr{V}_{h, 0}\right)_{t} \equiv L^{2}\left(0, T ; \mathscr{V}_{h}\right)$ and $\left(\mathscr{Q}_{h}\right)_{t} \equiv L^{2}\left(0, T ; Q_{h}\right)$. Existence and uniqueness of the semi-discrete system (13) are known.

In order to consider the time discretization of system (13) some extra notation is needed. Given a Banach space $X$ equipped with $\|\cdot\|_{X}$, a continuous function $f:[0, T] \rightarrow X$ and two real numbers $1 \leqslant p<\infty$ and $\alpha>0$, for a time step size $\delta t>0$, let $t^{n}=n \delta t$ for $n=0, \ldots, N=[T / \delta t]$. Let $\ell^{p}(X)$ be the space of sequences $\left\{f^{n+1}\right\}_{n=0}^{N-1}$ such that $\left(\sum_{n=0}^{N-1} \delta t\left\|f^{n+1}\right\|_{X}^{p}\right)^{1 / p}<\infty$, and $\ell^{\infty}(X)$ the space of sequences such that $\sup _{n=0, \ldots, N-1}\left\|f^{n+1}\right\|_{X}<\infty$. From here onwards we denote by $N=[T / \delta t]-1$. We also define the difference operators:

$$
\delta f^{n+1}=f^{n+1}-f^{n}, \quad \delta_{t} f^{n+1}=\frac{1}{\delta t} \delta f^{n+1} .
$$

For the time discretization of (13) we consider a backward Euler scheme. Nevertheless, we will discuss the extension of the numerical analyses to second order backward differencing (BDF2) and Crank-Nicholson time integrators.

\section{The variational multiscale anproach}

\subsection{The stationary Stokes problem}

In this section, we recall the variational multiscale approach to stabilized methods initially suggested by Hughes in $[19,20]$. This framework is useful for the motivation of the dynamic subscales method analyzed in this article.

Let us start rewriting the stationary Stokes system (7) in the following abstract form: find $U \in \mathscr{W}$ such that: $B(U, V)=L(V) \quad \forall V \in \mathscr{W}$

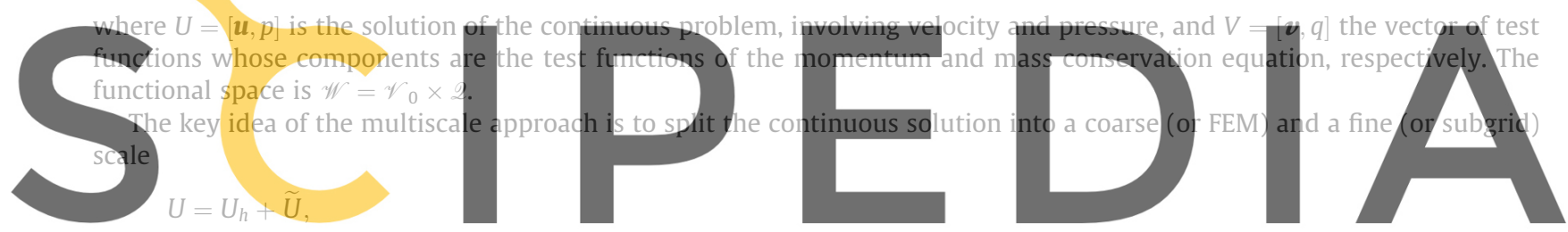

where $U_{h} \in \mathscr{W}_{h}=\mathscr{V}_{h .0} \times \mathscr{Q}_{h}$ and $\widetilde{U} \in \widetilde{\mathscr{W}}$, the subgrid space. In order to have a unique decomposition, we assume that

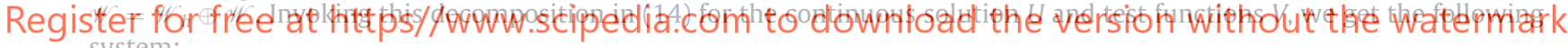
system:

$$
\begin{aligned}
& B\left(U_{h}+\widetilde{U}, V_{h}\right)=L\left(V_{h}\right) \quad \forall V_{h} \in \mathscr{W}_{h}, \\
& B\left(U_{h}+\widetilde{U}, \widetilde{V}\right)=L(\widetilde{V}) \quad \forall \widetilde{V} \in \widetilde{\mathscr{W}} .
\end{aligned}
$$

To solve this problem is as difficult as the original one. At this point, further simplification are required, in order to get a computationally feasible numerical method. From the subgrid equation (15b), we motivate a simplified expression for the subgrid component in terms of the finite element component, $\widetilde{U} \simeq \widetilde{U}\left(U_{h}\right)$. This approximation is known as the modelling for the subscales. In order to motivate this subgrid model, we integrate by parts some terms of the subgrid equation (15b) and neglect inter-element jumps terms, getting:

$$
\langle\mathscr{L}(\widetilde{U}), \widetilde{V}\rangle=\left\langle F-\mathscr{L}\left(U_{h}\right), \widetilde{V}\right\rangle,
$$

where $\mathscr{L}$ is the Stokes differential operator:

$$
\mathscr{L}(U)=\left(\begin{array}{c}
-v \Delta \boldsymbol{u}+\nabla p \\
\nabla \cdot \boldsymbol{u}
\end{array}\right) .
$$

We can rewrite (16) as

$$
\Pi_{\widetilde{W}}(\mathscr{L}(\widetilde{U}))=\Pi_{\widetilde{W}}\left(F-\mathscr{L}\left(U_{h}\right)\right),
$$

where $\Pi \sim$ is the $L^{2}$-projection onto $\widetilde{\mathscr{W}}$ and the residual of the finite element component is at the right hand side of (17). This kind of methods are known as residual-based.

In order to get a feasible numerical method, some approximations are made. The subgrid component is localized to the element by replacing the Stokes operator by the element-by-element Stokes operator $\mathscr{L}_{h}$ where the Laplacian $\Delta$ is replaced by the differential operator $\Delta_{h}$, used to indicate that the Laplacian is only evaluated on the interior of finite elements (not considering inter-element jumps). Furthermore, the inverse of $\Pi_{\widetilde{W}} \circ \mathscr{L}_{h}$ is approximated by an algebraic operator $\tau$, justified by Fourier analysis in [11]: 


$$
\tau=\operatorname{diag}\left\{\tau \boldsymbol{I}, \tau_{p}\right\}=\left(\begin{array}{cc}
C_{\tau} \frac{h^{2}}{v} \boldsymbol{I} & 0 \\
0 & v
\end{array}\right)
$$

where $\boldsymbol{I}$ is the identity matrix of dimension $d$ and $C_{\tau}$ is an algorithmic constant. The subgrid projection in the LHS of (17) is also approximated by the projection operator $\mathscr{P}(\cdot)$. After these three approximations, we end up with the following model for the subscales:

$$
\tau^{-1} \widetilde{U}=\mathscr{P}\left(F-\mathscr{L}_{\hbar}\left(\mathscr{U}_{\hbar}\right)\right) .
$$

The operator $\mathscr{P}(\cdot)$ varies with the stabilized method chosen. For ASGS this operator is the $L^{2}$-identity, denoted by $\mathscr{I}(\cdot)$ (see e.g. [19]). On the other hand, if we consider the orthogonal subscales stabilization (OSS) developed e.g. in [11], the $L^{2}$ orthogonal projection onto the finite element space for the velocity is used:

$$
\mathscr{P}(\cdot)=\Pi_{h}^{\perp}(\cdot)=\mathscr{I}(\cdot)-\Pi_{h}(\cdot),
$$

where $\Pi_{h}(\cdot)$ denotes the $L^{2}$ projection onto the finite element space $\mathscr{V}_{h}$.

Remark 3.1. Using OSS we satisfy $\widetilde{\mathscr{W}} \cap \mathscr{W}_{h}=\{0\}$ but not with ASGS. The violation of this property makes ASGS more diffusive and affects negatively the stability properties of the finite element component, as proved in the next section. We refer to Codina and Blasco [13] for numerical experiments that evidence this behavior.

Invoking the expression for the subgrid component at the finite element equation (15a) and integrating by parts those terms related to the subgrid component we get:

$$
B\left(U_{h}+\widetilde{U}\left(U_{h}\right), V_{h}\right) \simeq B\left(U_{h}, V_{h}\right)+\left\langle\widetilde{U}\left(U_{h}\right), \mathscr{L}_{h}^{*}\left(V_{h}\right)\right\rangle:=B_{h}\left(U_{h}, V_{h}\right),
$$

where again we neglect inter-element jumps. $\mathscr{L}_{h}^{*}$ is the formal adjoint of the Stokes operator evaluated elementwise. Therefore, we have obtained a modified bilinear form $B_{h}$ only in terms of the finite element component. The point is to check that the inf-sup condition for the stabilized problem:

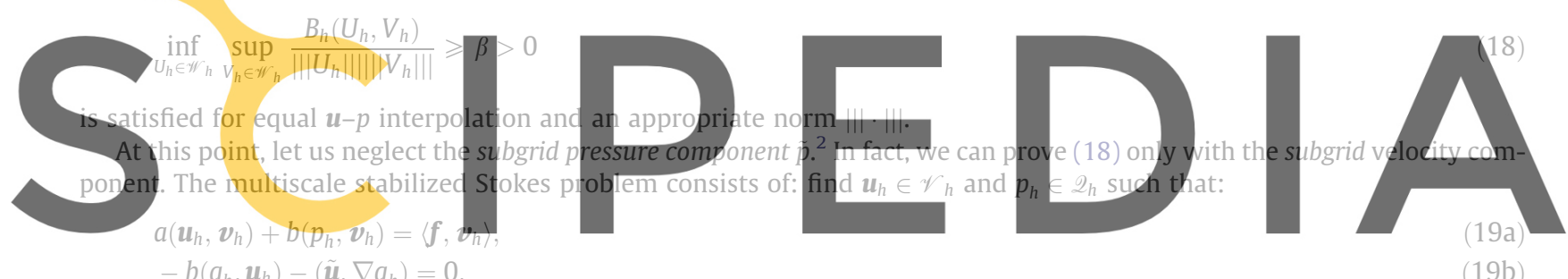

Register for free at https/fwysuyscipedia,com to download the version without the watermark

$$
\tau^{-1} \tilde{\boldsymbol{u}}=\mathscr{P}\left(\boldsymbol{f}+v \Delta_{h} \boldsymbol{u}_{h}-\nabla p_{h}\right) .
$$

Remark 3.2. It is clear that $v \Delta_{h} \boldsymbol{u}_{h}$ is identically zero for linear elements. On the other hand, this term is needed in order to keep optimal convergence when using high order finite elements and ASGS. Nevertheless, for OSS this term can be neglected in any case without loosing order of convergence, as proved in Section 5.

Remark 3.3. The orthogonal subscales method can also be considered non-residual based due to the fact that it keeps optimal convergence even taking out finite element terms in (20) (under appropriate regularity properties) that do not play a key role on stabilization, like the diffusive or force terms.

\subsection{The transient Stokes problem}

Let us start writing the transient Stokes problem in an abstract setting:

$$
\left(D_{t} U, V\right)+B(U, V)=L(V) \quad \forall V \in \mathscr{W},
$$

where $D_{t} U=\left[\partial_{t} \boldsymbol{u}, 0\right]$. Now, $U(t)=[\boldsymbol{u}(t), p(t)]$ belongs to $\mathscr{W}_{t}=\left(\mathscr{V}_{0}\right)_{t} \times \mathscr{Q}_{t}$. Again, we decompose this solution into $U_{h} \in\left(\mathscr{W}_{h}\right)_{t} \equiv\left(\mathscr{V}_{h, 0}\right)_{t} \times\left(\mathscr{Q}_{h}\right)_{t}$ and $\widetilde{U} \in \widetilde{W}_{t}$, such that $\mathscr{W}_{t}=\left(\mathscr{W}_{h}\right)_{t} \oplus \widetilde{\mathscr{W}}_{t}$. Invoking this decomposition in (21), we get:

\footnotetext{
${ }^{2}$ This is a reasonable simplification because the pressure subgrid scale only gives stability over the velocity. This stability is not needed since the velocity is already controlled by the Galerkin terms. We refer to Codina [11] for the obtention of the subgrid pressure stabilization terms. It is interesting to note that in other problems in which velocity stabilization is needed too, like Darcy flow, this pressure subscale plays an important role (see [2]).
} 


$$
\begin{aligned}
& \left(D_{t} U_{h}, V_{h}\right)+\left(D_{t} \widetilde{U}, V_{h}\right)+B\left(U_{h}, V_{h}\right)+B\left(\widetilde{U}, V_{h}\right)=L\left(V_{h}\right), \\
& \left(D_{t} \widetilde{U}, \widetilde{V}\right)+\left(D_{t} U_{h}, \widetilde{V}\right)+B(\widetilde{U}, \widetilde{V})+B\left(U_{h}, \widetilde{V}\right)=L(\widetilde{V}) .
\end{aligned}
$$

Following the procedure used for the stationary case, that is, integrating by parts, neglecting inter-element jump terms and localizing the subgrid equation to the element level in (22), we obtain:

$$
\begin{aligned}
& \left(D_{t} U_{h}, V_{h}\right)+\left(D_{t} \widetilde{U}, V_{h}\right)+B\left(U_{h}, V_{h}\right)+\left\langle\widetilde{U}, \mathscr{L}_{h}^{*}\left(V_{h}\right)\right\rangle=L\left(V_{h}\right), \\
& \left(D_{t} \widetilde{U}, \widetilde{V}\right)+\left(D_{t} U_{h}, \widetilde{V}\right)+\left\langle\mathscr{L}_{h}(\widetilde{U}), \widetilde{V}\right\rangle+\left\langle\mathscr{L}_{h}\left(U_{h}\right), \widetilde{V}\right\rangle=L(\widetilde{V}) .
\end{aligned}
$$

As for the stationary case, we approximate the Stokes differential operator by an algebraic operator $\tau$ and replace the subgrid projection by the identity (for ASGS) or the orthogonal projection onto the finite element space (for OSS). Nevertheless, in (23b) it does appear a new term, the time derivative of the subgrid component, that is considered as such, getting the following ordinary differential equation:

$$
D_{t} \widetilde{U}+\tau^{-1} \widetilde{U}=\mathscr{P}\left(F-\mathscr{L}_{h}\left(U_{h}\right)-D_{t} U_{h}\right) .
$$

This new methodology, coined in [15] as dynamic subscales, turns out to have much better stability properties than the quasistatic approximation, as proved in the next section.

The tracking of the subscales in time is not standard at all. The standard quasi-static methodology used for stabilizing the transient problem is based on the following subgrid model:

$$
\tau_{q S}^{-1} \widetilde{U}=\mathscr{P}\left(F-\mathscr{L}_{h}\left(U_{h}\right)-D_{t} U_{h}\right) .
$$

The straightforward expression for the stabilization parameter is to take $\tau_{q s}=\tau$, motivated by neglecting the subgrid time derivative in (23b) (by the quasi-static assumption) and approximating $\mathscr{L}_{h}$ as above. Another approach is to approximate the time derivative term in (23b) by a reaction-like term (with a zero order derivative) that introduces a factor $1 / \delta t$ in the stabilization parameter (see [22,17]):
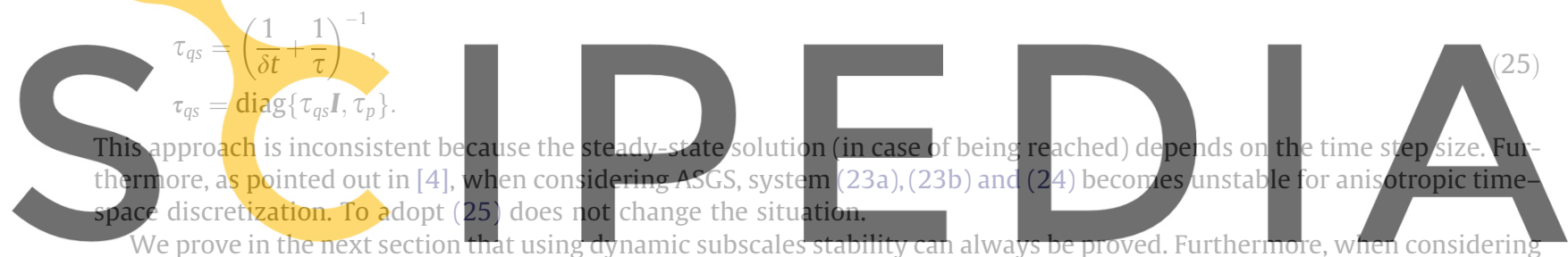

OSS for the subgrid scale model, stability is kept even for quasi-static subscales, using as stabilization term $\tau_{q s}$, under mild

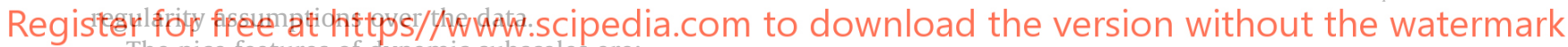
The nice features of dynamic subscales are:

- The steady-state case does not depend on $\delta t$ anymore.

- We can prove stability without any condition over the time-space discretization and minimum regularity assumptions over the data.

- Both ASGS and OSS subscale choices for the projection lead to stable methods.

Neglecting the subgrid pressure again, we can write the multiscale stabilized transient Stokes system. We introduce the parameter $\gamma$ that takes the value 1 for dynamic subscales and 0 for quasi-static subscales. The problem reads as: find $\boldsymbol{u}_{h}(t) \in L^{2}\left(0, T ; \mathscr{V}_{h}\right)$ and $p_{h}(t) \in L^{2}\left(0, T ; \mathscr{Q}_{h}\right)$ such that:

$$
\begin{aligned}
& \left(\partial_{t} \boldsymbol{u}_{h}(t), \boldsymbol{v}_{h}\right)+v\left(\nabla \boldsymbol{u}_{h}(t), \nabla \boldsymbol{v}_{h}\right)-\left(p_{h}(t), \nabla \cdot \boldsymbol{v}_{h}\right)+\gamma\left(\partial_{t} \tilde{\boldsymbol{u}}(t), \boldsymbol{v}_{h}\right)=\left\langle\boldsymbol{f}(t), \boldsymbol{v}_{h}\right\rangle, \\
& \left(q_{h}, \nabla \cdot \boldsymbol{u}_{h}(t)\right)-\left(\tilde{\boldsymbol{u}}(t), \nabla q_{h}\right)=0,
\end{aligned}
$$

where the subgrid component is obtained from the following equation:

$$
\gamma \partial_{t} \tilde{\boldsymbol{u}}(t)+\tau^{-1} \tilde{\boldsymbol{u}}(t)=\mathscr{P}\left(\boldsymbol{f}(t)-\nabla p_{h}(t)-\partial_{t} \boldsymbol{u}_{h}(t)\right) .
$$

This system is initialized with $\boldsymbol{u}_{h}^{0}=\Pi_{R}\left(\boldsymbol{u}^{0}\right)$ (where $\Pi_{R}$ is the Riesz projector used in [10]) and $\tilde{\boldsymbol{u}}^{0}=0$. Using the Riesz projector, $\boldsymbol{u}_{h}^{0}$ satisfies (26b) at $t=0$, a property that is needed in the following numerical analysis (see Remark 4.8). We neglect the Laplacian term in (27) for the sake of simplicity. How the introduction of this term affects the results is explained in Remark 4.7. This term must be introduced for high order elements together with ASGS in order to keep the appropriate order of convergence.

Let us summarize the different stabilization methods considered in this article:

- $\gamma=1$ and $\mathscr{P}=\Pi_{h}^{\perp}$ : dynamic orthogonal subscales (D-OSS).

- $\gamma=1$ and $\mathscr{P}=\mathscr{I}$ : dynamic algebraic subscales (D-ASGS). 
Table 1

List of main results.

\begin{tabular}{lll}
\hline Method & Main result & Label \\
\hline D-OSS & Stability (semi-discrete) \\
& Stability (fully discrete) & Corollary 4.2 \\
Theorem 5.1 & Convergence \\
D-ASGS & Stability (semi-discrete) \\
& Stability (fully discrete) \\
Q-OSS & Stability (semi-discrete) \\
Q-ASGS & Stability (fully discrete) \\
\hline
\end{tabular}

- $\gamma=0$ and $\mathscr{P}=\Pi_{h}^{\perp}$ : quasi-static orthogonal subscales (Q-OSS).

- $\gamma=0$ and $\mathscr{P}=\mathscr{I}$ : quasi-static algebraic subscales (Q-ASGS).

In Table 1, we provide the reader with a road map for the subsequent analysis.

Remark 3.4. In this work, we will show the superiority (for ASGS) of dynamic subscales with respect to quasi-static subscales using stability arguments. However, the benefits of using dynamic subscales are not restricted to stability arguments; they have advantages from the point of view of the time integration, solve the conflict about the design of the stabilization terms for time dependent problems (either at the semi-discrete or the fully discrete level) and the numerical experiments show that the gain with respect to quasi-static subscales is notorious (see [15]). Furthermore, it has been proved in [24] that dynamic subscales stabilization behaves as a turbulence model that allows backscatter (not possible for quasi-static subscales).

\section{Stability results for the transient Stokes problem}

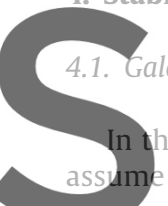

\section{Galerkin approximation}

In this section, we consider the that a discreteinf-sup confition hol that a discrete inf-sup cor
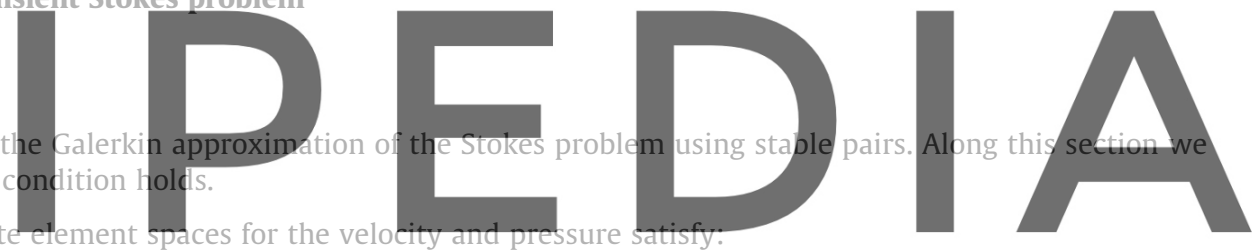

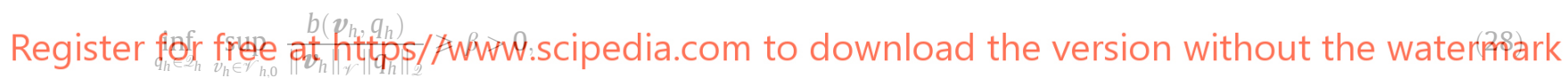

with $\beta$ uniform with respect to the mesh size $h$.

Let us consider the semi-discrete (in space) Stokes problem in its weak form: find $\boldsymbol{u}_{h}(t) \in L^{2}\left(0, T ; \mathscr{V}_{h}\right)$ and $p_{h}(t) \in L^{2}\left(0, T ; \mathscr{2}_{h}\right)$ such that:

$$
\begin{aligned}
& \left(\partial_{t} \boldsymbol{u}_{h}(t), \boldsymbol{v}_{h}\right)+v\left(\nabla \boldsymbol{u} h(t), \nabla \boldsymbol{v}_{h}\right)-\left(p_{h}(t), \nabla \cdot \boldsymbol{v}_{h}\right)=\left\langle\boldsymbol{f}(t), \boldsymbol{v}_{h}\right\rangle, \\
& \left(q_{h}, \nabla \cdot \boldsymbol{u}_{h}(t)\right)=0
\end{aligned}
$$

initialized with $\boldsymbol{u}_{h}^{0}=\Pi_{R}\left(\boldsymbol{u}^{0}\right)$. This system is a differential-algebraic (DAE) system. Therefore, its solution $\left\{\boldsymbol{u} h(t), p_{h}(t)\right\}$ does exist, is unique and continuous in time. The proof of the stability bounds stated in the next theorem can be found in [26]. In order to get these stability results, strong regularity assumptions over the data are needed; that is to say, more regularity than the one needed for the problem to be well-posed.

Theorem 4.1. Given a force vector $\boldsymbol{f}(t) \in L^{2}\left(0, T ; \boldsymbol{L}^{2}(\Omega)\right)$ and initial condition $\boldsymbol{u}^{0} \in \boldsymbol{H}_{0}^{1}(\Omega)$, the solution $\left\{\boldsymbol{u}_{h}(t), p_{h}(t)\right\}$ of system (29) satisfies:

$$
\begin{aligned}
& \boldsymbol{u}_{h}(t) \in L^{\infty}\left(0, T ; \boldsymbol{L}^{2}(\Omega)\right) \cap L^{2}\left(0, T ; \boldsymbol{H}_{0}^{1}(\Omega)\right), \\
& \partial_{t} \boldsymbol{u}_{h}(t) \in L^{2}\left(0, T ; \boldsymbol{L}^{2}(\Omega)\right), \\
& p_{h}(t) \in L^{2}\left(0, T ; L^{2}(\Omega)\right) .
\end{aligned}
$$

Remark 4.1. We can get a much weaker pressure stability result under minimum regularity assumptions over the force term and initial velocity (see [26]), which is

$$
\left\|\int_{0}^{T} p_{h}(s) d s\right\|_{0} \leqslant C
$$


Now we want to obtain similar results for the fully discretized version of (29). Let us write the fully discretized Stokes problem in its weak form. For the sake of simplicity, we consider the backward Euler time integration scheme. For a given time step value $t^{n+1}$, the problem consists of finding $\boldsymbol{u}_{h}^{n+1} \in \mathscr{V}_{h}$ and $p_{h}^{n+1} \in \mathscr{Q}_{h}$ such that ${ }^{3}$ :

$$
\begin{aligned}
& \left(\delta_{t} \boldsymbol{u}_{h}^{n+1}, \boldsymbol{v}_{h}\right)+v\left(\nabla \boldsymbol{u}_{h}^{n+1}, \nabla \boldsymbol{v}_{h}\right)-\left(p_{h}^{n+1}, \nabla \cdot \boldsymbol{v}_{h}\right)=\left\langle\boldsymbol{f}^{n+1}, \boldsymbol{v}_{h}\right\rangle, \\
& \left(q_{h}, \nabla \cdot \boldsymbol{u}_{h}^{n+1}\right)=0 .
\end{aligned}
$$

We summarize the stability for the discrete Stokes problem (30) in the following corollary. We omit the proof because it is analogous to the one for the semi-discrete problem.

Corollary 4.1. Given a force vector $\left\{\boldsymbol{f}^{n+1}\right\}_{n=0}^{N-1} \in \ell^{2}\left(\boldsymbol{L}^{2}(\Omega)\right)$ and initial condition $\boldsymbol{u}^{0} \in \boldsymbol{H}_{0}^{1}(\Omega)$, the sequence $\left\{\boldsymbol{u}_{h}^{n+1}, p_{h}^{n+1}\right\}_{n=0}^{N-1}$ of solutions of system (29) satisfies:

$$
\begin{aligned}
& \left\{\boldsymbol{u}_{h}^{n+1}\right\}_{n=0}^{N-1} \in \ell^{\infty}\left(\boldsymbol{L}^{2}(\Omega)\right) \cap \ell^{2}\left(\boldsymbol{H}_{0}^{1}(\Omega)\right), \\
& \left\{\delta_{t} \boldsymbol{u}_{h}^{n+1}\right\}_{n=0}^{N-1} \in \ell^{2}\left(\boldsymbol{L}^{2}(\Omega)\right), \\
& \left\{p_{h}^{n+1}\right\}_{n=0}^{N-1} \in \ell^{2}\left(L^{2}(\Omega)\right) .
\end{aligned}
$$

Remark 4.2. The stability results in Corollary 4.1 have been obtained without taking benefit of the numerical dissipation of the time integration scheme, since we have pursued the proof for the semi-discrete problem. Therefore, these results are valid for any $\mathscr{A}$-stable time integration scheme, like backward Euler, Crank-Nicholson and BDF2.

Remark 4.3. Again, we can get weaker stability bounds under minimum regularity assumptions, that is, $\left\{\boldsymbol{f}^{n}\right\}_{n=0}^{N-1} \in \ell^{2}\left(\boldsymbol{H}^{-1}(\Omega)\right)$ and $\boldsymbol{u}^{0} \in \mathbf{L}^{2}(\Omega)$, obtaining:
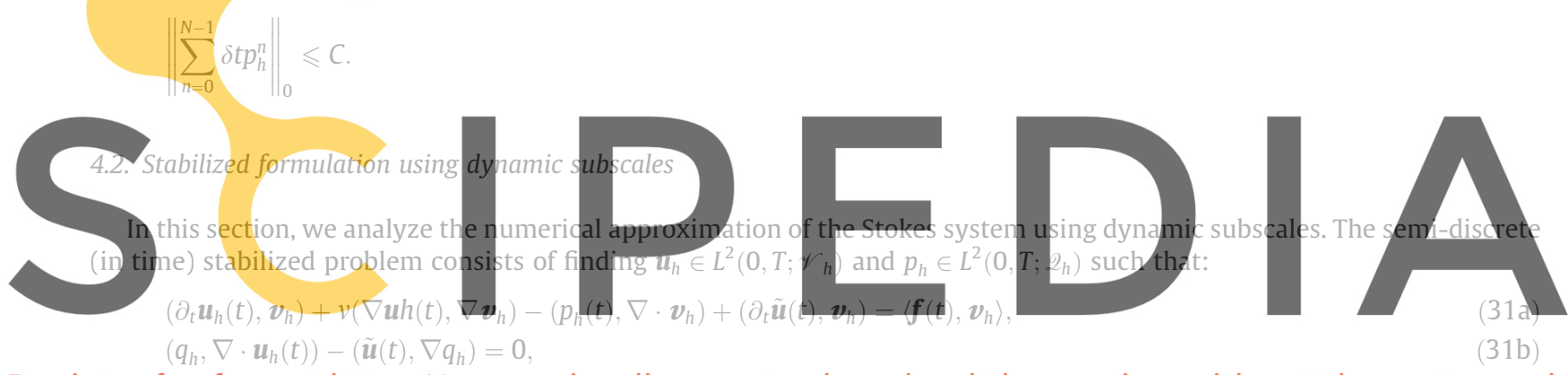

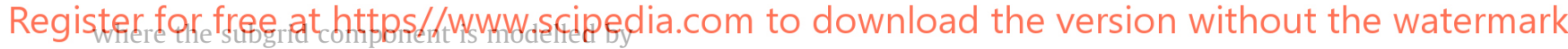

$$
\partial_{t} \tilde{\boldsymbol{u}}(t)+\tau^{-1} \tilde{\boldsymbol{u}}(t)=\mathscr{P}\left(\boldsymbol{f}(t)-\nabla p_{h}(t)-\partial_{t} \boldsymbol{u}_{h}(t)\right) .
$$

We have analyzed the semi-discrete (in time) and fully discrete versions.

Along this section the following assumption will be used.

Assumption 2. The family of finite element partitions $\left\{\Theta_{h}\right\}_{h>0}$ is quasi-uniform.

This assumption is needed because if it holds the following inverse estimate (see [7]) can be used: given $\boldsymbol{v}_{h} \in \mathscr{V}_{h, 0}$, there exists a constant $C_{h}$ independent of the mesh size $h$ such that:

$$
\left\|\boldsymbol{v}_{h}\right\|_{1} \leqslant \frac{C_{h}}{h}\left\|\boldsymbol{v}_{h}\right\|_{0}
$$

The quasi-uniformity can be relaxed, assuming only non-degenerate finite element partitions (see [14]). The key idea is to replace (33) by local inverse inequalities.

The stabilized Stokes system, motivated from a multiscale approach, has already been stated in (26b) and (27). Let us remark one important feature of this system. It is true that our finite element velocity $\boldsymbol{u}_{h}(t)$ is not divergence-free. However, from (26b), we can easily infer that $\boldsymbol{u}_{h}+\tilde{\boldsymbol{u}}$ is solenoidal in a particular sense.

In the next theorem we state some stability bounds of system (31) and (32). The strategy of the proof is split into two parts. In a first step stability bounds have been obtained not only for the finite element velocity, but also the subgrid component. After that, we translate this subgrid stability in terms of pressure stability. This proof is non-standard. Standard sta-

\footnotetext{
${ }^{3}$ If the body force $\boldsymbol{f}$ is not continuous in time, we simply define$$
\boldsymbol{f}^{n+1}:=\int_{t^{n}}^{t^{n+1}} \boldsymbol{f}(t) d t
$$ 
bilized methods are only written in terms of the finite element component because the subgrid component has a closed form in terms of the finite element component. We cannot use this approach for dynamic subscales because the subgrid equation is a differential equation.

We need to use extra notation. Given a functional space $X$ and a mesh dependent scalar value $\tau>0$, we will say that a function $u$ belongs to $\tau X$ if $\tau u$ belongs to $X$.

Theorem 4.2. Given a force vector sequence $\boldsymbol{f}(t) \in L^{2}\left(0, T ; \boldsymbol{H}^{-1}(\Omega)\right) \cap L^{2}\left(0, T ; \tau^{1 / 2} \boldsymbol{L}^{2}(\Omega)\right)$ and initial condition $\boldsymbol{u}^{0} \in \boldsymbol{L}^{2}(\Omega)$, the solution $\left\{\boldsymbol{u} h(t), p_{h}(t)\right\}$ of system (31) and (32) with $\mathscr{P}(\cdot)=\mathscr{I}(\cdot)$ satisfies:

$$
\begin{aligned}
& \boldsymbol{u}_{h}(t) \in L^{2}\left(0, T ; \boldsymbol{H}_{0}^{1}(\Omega)\right), \\
& \nabla p_{h}(t) \in L^{2}\left(0, T ; \tau^{1 / 2} \mathbf{L}^{2}(\Omega)\right) .
\end{aligned}
$$

Under the same hypothesis, with $\mathscr{P}(\cdot)=\Pi_{h}^{\perp}(\cdot)$, the stability results are:

$$
\begin{aligned}
& \boldsymbol{u}_{h}(t) \in L^{\infty}\left(0, T ; \boldsymbol{L}^{2}(\Omega)\right) \cap L^{2}\left(0, T ; \boldsymbol{H}_{0}^{1}(\Omega)\right), \\
& \partial_{t} \boldsymbol{u}_{h}(t) \in L^{2}\left(0, T ; \tau^{1 / 2} \mathbf{L}^{2}(\Omega)\right), \\
& \nabla p_{h}(t) \in L^{2}\left(0, T ; \tau^{1 / 2} \boldsymbol{L}^{2}(\Omega)\right) .
\end{aligned}
$$

Proof. Let us start rewriting (31a) and (32) in a more appropriate version for the subsequent analysis. Grouping time derivatives we have:

$$
\begin{aligned}
& \left(\partial_{t}\left(\boldsymbol{u}_{h}+\tilde{\boldsymbol{u}}\right)(\boldsymbol{t}), \boldsymbol{v}_{h}\right)+v\left(\nabla \boldsymbol{u} h(t), \nabla \boldsymbol{v}_{h}\right)-\left(p_{h}(t), \nabla \cdot \boldsymbol{v}_{h}\right)=\left\langle\boldsymbol{f}(t), \boldsymbol{v}_{h}\right\rangle, \\
& \partial_{t}\left(\mathscr{P}\left(\boldsymbol{u}_{h}\right)+\tilde{\boldsymbol{u}}\right)(\boldsymbol{t})+\tau^{-1} \tilde{\boldsymbol{u}}(t)=\mathscr{P}\left(\boldsymbol{f}(t)-\nabla p_{h}(t)\right) .
\end{aligned}
$$

We can obtain (FEM and subgrid) velocity bounds using the following strategy. First, we test (34a) and (31b) with $\boldsymbol{v}_{h}=\boldsymbol{u}_{h}(t)$ and $q_{h}=p_{h}(t)$, respectively, for every time instant $t \in(0, T)$. After that, we multiply (34b) by $\tilde{\boldsymbol{u}}(t)$, and integrate over the domain $\Omega$. Adding up these equations, and integrating over the whole time domain $(0, T)$, we get:
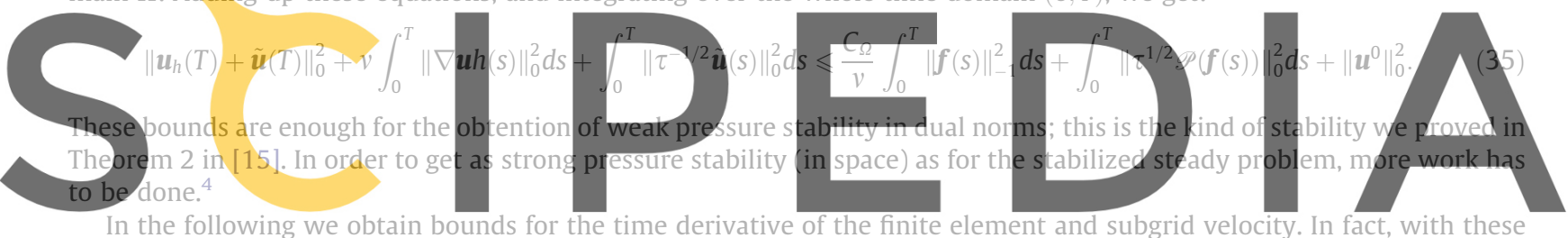

bounds we can improve the stability results in [15] making the translation process much more natural We test (34a) with Registerffor, direeniag: https//www.scipedia.com to download the version without the watermark

$$
\left(\partial_{t}\left(\boldsymbol{u}_{h}+\tilde{\boldsymbol{u}}\right)(t), \partial_{t} \boldsymbol{u}_{h}(t)\right)+v\left(\nabla \boldsymbol{u}_{h}(t), \nabla \partial_{t} \boldsymbol{u}_{h}(t)\right)-\left(p_{h}(t), \nabla \cdot \partial_{t} \boldsymbol{u}_{h}(t)\right)=\left\langle\boldsymbol{f}(t), \partial_{t} \boldsymbol{u}_{h}(t)\right\rangle
$$

Now, in order to bound the last term of the left hand side, let us manipulate the subgrid equation. We multiply (32) by $\partial_{t} \tilde{\boldsymbol{u}}$ and integrate the result over the whole domain $\Omega$, getting:

$$
\left(\partial_{t}\left(\boldsymbol{u}_{h}+\tilde{\boldsymbol{u}}\right)(t), \partial_{t} \tilde{\boldsymbol{u}}(t)\right)+\left(\tau^{-1} \tilde{\boldsymbol{u}}(t), \partial_{t} \tilde{\boldsymbol{u}}(t)\right)=\left(\mathscr{P}\left(\boldsymbol{f}(t)-\nabla p_{h}(t)\right), \partial_{t} \tilde{\boldsymbol{u}}\right) .
$$

Taking the time derivative of (31b), and evaluating the derived equation with $q_{h}=p_{h}(t)$, we obtain:

$$
\left(p_{h}(t), \nabla \cdot \partial_{t} \boldsymbol{u}_{h}(t)\right)-\left(\partial_{t} \tilde{\boldsymbol{u}}(t), \nabla p_{h}(t)\right)=0 .
$$

Adding up (36)-(38), multiplying by $\tau$ and integrating in time the resulting equation, we get:

$$
\int_{0}^{T}\left\|\tau^{1 / 2} \partial_{t}\left(\boldsymbol{u}_{h}+\tilde{\boldsymbol{u}}\right)(s)\right\|_{0}^{2} d s+v\left\|\tau^{1 / 2} \nabla \boldsymbol{u}_{h}(T)\right\|_{0}^{2}+\|\tilde{\boldsymbol{u}}(T)\|_{0}^{2} \leqslant \int_{0}^{T}\left\|\tau^{1 / 2} \boldsymbol{f}(s)\right\|_{0}^{2} d s+C_{h} C_{\tau}\left\|\boldsymbol{u}_{h}^{0}\right\|_{0}^{2}+\left\|\tilde{\boldsymbol{u}}^{0}\right\|_{0}^{2},
$$

where we have used a classical inverse estimate and the definition of the stabilization parameter in the term related to the initial finite element velocity $\boldsymbol{u}_{h}^{0}$. Let us point out that the subgrid term in (38) is canceled with the RHS of (37) due to the fact that $\left(\mathscr{P}\left(\nabla p_{h}(t)\right), \partial_{t} \tilde{\boldsymbol{u}}\right)=\left(\nabla p_{h}(t), \partial_{t} \tilde{\boldsymbol{u}}\right)$.

Now, we translate the subgrid stability in terms of finite element stability. Let us start by multiplying (34b) by $\tau^{1 / 2}$ and taking its $L^{2}(\Omega)$ norm. Integrating the resulting equation over the whole time domain we get:

$$
\frac{1}{3} \int_{0}^{T}\left\|\tau^{1 / 2} \mathscr{P}\left(\nabla p_{h}(s)\right)\right\|_{0}^{2} d s \leqslant \int_{0}^{T}\left\|\tau^{1 / 2} \partial_{t}\left(\mathscr{P}\left(\boldsymbol{u}_{h}\right)+\tilde{\boldsymbol{u}}\right)(s)\right\|_{0}^{2} d s+\int_{0}^{T}\left\|\tau^{-1 / 2} \tilde{\boldsymbol{u}}(s)\right\|_{0}^{2} d s+\int_{0}^{T}\left\|\tau^{1 / 2} \mathscr{P}(\boldsymbol{f}(s))\right\|_{0}^{2} d s .
$$

\footnotetext{
${ }^{4}$ This is the essence of this work, to obtain the same pressure stability (in space) for the stabilized transient problem as for the steady one without any assumption over the time step size.
} 
At this point, we only have stability over the orthogonal projection of the pressure gradient $\Pi_{h}^{\perp}\left(\nabla p_{h}\right)$. A further step is needed; we test (34a) for a given time instant $t$ with $\boldsymbol{v}_{h}=\Pi_{h}\left(\nabla p_{h}(t)\right)$ and use a classical inverse inequality for the viscous term at the right hand side, obtaining:

$$
\left\|\Pi_{h}\left(\nabla p_{h}(t)\right)\right\|_{0}^{2} \leqslant\left\|\Pi_{h}\left(\nabla p_{h}(t)\right)\right\|_{0} \times\left\{\left\|\Pi_{h}(\boldsymbol{f}(t))\right\|_{0}+\left\|\partial_{t}\left(\boldsymbol{u}_{h}+\tilde{\boldsymbol{u}}\right)(t)\right\|_{0}+\frac{C_{h} v}{h}\left\|\nabla \boldsymbol{u}_{h}(t)\right\|_{0}\right\} .
$$

Multiplying the previous equation by $\tau$ and using its expression, squaring both terms of the resulting inequality and integrating in time, we get a bound for $\Pi_{h}\left(\nabla p_{h}\right)$. Combining the previous bounds we get the stability over $\nabla p_{h}$ in the theorem.

Remark 4.4. In order to simplify the exposition, we are assuming that $\tau$ is constant in space. However, we could also consider $\tau$ space dependent (see [12]).

Remark 4.5. In the previous theorem we can see the impact of $\mathscr{P}(\cdot)$ on the stability results. When using ASGS, the operator is $\mathscr{P}(\cdot)=\mathscr{I}(\cdot)$. We are violating the fact that, without any approximation, finite element and subgrid spaces are complimentary. Even though optimal results are obtained over $\boldsymbol{u}_{h}+\tilde{\boldsymbol{u}}$, we cannot recover some stability estimates over $\boldsymbol{u}_{h}$. However, with $\mathscr{P}(\cdot)=\Pi_{h}^{\perp}(\cdot)$ finite element component and subgrid component are orthogonal. Therefore, we know that $\left\|\boldsymbol{u}_{h}+\tilde{\boldsymbol{u}}\right\|_{0}^{2}=\left\|\boldsymbol{u}_{h}\right\|_{0}^{2}+\|\tilde{\boldsymbol{u}}\|_{0}^{2}$, and we can recover for the FEM component stability estimates similar to those obtained under the inf-sup condition. Nevertheless, even though we have not included this stability in the theorem thesis because it involves the subgrid component, we have also got $\boldsymbol{u} h(t)+\tilde{\boldsymbol{u}}(t) \in L^{\infty}\left(0, T ; \boldsymbol{L}^{2}(\Omega)\right)$ and $\partial_{t}\left(\boldsymbol{u}_{h}(t)+\tilde{\boldsymbol{u}}(t)\right) \in L^{2}\left(0, T ; \tau^{1 / 2} \boldsymbol{L}^{2}(\Omega)\right)$.

Remark 4.6. The assumption over the force term can be simply reduced to $\boldsymbol{f}(t) \in L^{2}\left(0, T ; \boldsymbol{H}^{-1}(\Omega)\right)$ given $\boldsymbol{f}(t) \in \mathscr{V}_{h}$ for all time instants. This can be proved using inverse estimates and the definition of the stabilization parameter. Using OSS and neglecting the force term in (32) (see Remark 3.3) we can also get these results under minimum regularity over the force term.

Remark 4.7. In case of considering the Laplacian of the subgrid component, some extra considerations must be addressed. In
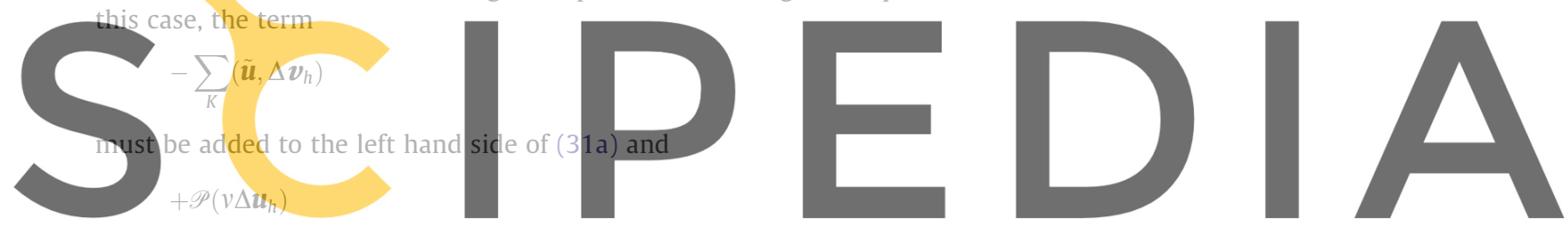

to the right hand side of (32). In this case, for the obtention of (35) the following term must be controlled:

Register for free at https//www.scipedia.com to download the version without the watermark $-2 \sum_{K}\left(\tilde{\boldsymbol{u}}, v \Delta \boldsymbol{u}_{h}\right)_{K} \leqslant \frac{1}{2}\left\|\tau^{-1 / 2} \tilde{\boldsymbol{u}}\right\|_{0}^{2}+4 C_{h}^{2} C_{\tau} v\left\|\nabla \boldsymbol{u}_{h}\right\|_{0}^{2}$.

Similarly for the obtention of (39). Therefore, in order to recover the results of Theorem 4.2, the stabilization parameter constant $C_{\tau}$ must satisfy:

$$
C_{\tau} \leqslant \frac{4}{v C_{h}^{2}}
$$

It can be checked that the same condition must be satisfied for the fully discrete problem and for quasi-static subscales.

From Theorem 4.2 it is seen we have recovered the same stability over the pressure than the one obtained using standard stabilization methods under the condition over the time step size (see Section 5). But now no conditions over time and space discretization are required. Thus, dynamic subscales deal well with anisotropic time-space discretizations. Let us remark that this is the first stability analysis of a residual-based stabilization technique obtained without relaying on condition (1) that gives pressure stability in strong norms (those for the stabilized problem in the steady case). The stability analysis in [15] was also true for any time step size, but the pressure stability was in very weak negative norms.

The stability properties of the time discrete version of (31) and (32) are analogous. Using the format of system (34b) and backward Euler for the time integration, the discrete problem is

$$
\begin{aligned}
& \left(\delta_{t}\left(\boldsymbol{u}_{h}^{n+1}+\tilde{\boldsymbol{u}}^{n+1}\right), \boldsymbol{v}_{h}\right)+v\left(\nabla \boldsymbol{u}_{h}^{n+1}, \nabla \boldsymbol{v}_{h}\right)-\left(p_{h}^{n+1}, \nabla \cdot \boldsymbol{v}_{h}\right)=\left\langle\boldsymbol{f}^{n+1}, \boldsymbol{v}_{h}\right\rangle, \\
& \left(q_{h}, \nabla \cdot \boldsymbol{u}_{h}^{n+1}\right)-\left(\tilde{\boldsymbol{u}}^{n+1}, \nabla q_{h}\right)=0, \\
& \delta_{t}\left(\mathscr{P}\left(\boldsymbol{u}_{h}^{n+1}\right)+\tilde{\boldsymbol{u}}^{n+1}\right)+\tau^{-1} \tilde{\boldsymbol{u}}^{n+1}=\mathscr{P}\left(\boldsymbol{f}^{n+1}-\nabla p_{h}^{n+1}\right) .
\end{aligned}
$$

The discrete counterpart of Theorem 4.2 can be proved in an analogous way. The stability properties of the stabilized discrete system when tracking the subscales in time are listed in the following corollary. 
Corollary 4.2. Given a force vector sequence $\left\{\boldsymbol{f}^{n+1}\right\}_{n=0}^{N-1} \in \ell^{2}\left(\boldsymbol{H}^{-1}(\Omega)\right) \cap \ell^{2}\left(\tau^{1 / 2} \mathbf{L}^{2}(\Omega)\right)$ and initial condition $\boldsymbol{u}^{0} \in \boldsymbol{L}^{2}(\Omega)$, the sequence of solutions $\left\{\boldsymbol{u}_{h}^{n+1}, p_{h}^{n+1}\right\}_{n=0}^{N-1}$ of system (41) with $\mathscr{P}(\cdot)=\mathscr{I}(\cdot)$ satisfies:

$$
\begin{aligned}
& \left\{\boldsymbol{u}_{h}^{n+1}\right\}_{n=0}^{N-1} \in \ell^{2}\left(\boldsymbol{H}_{0}^{1}(\Omega)\right), \\
& \left\{\nabla p_{h}^{n+1}\right\}_{n=0}^{N-1} \in \ell^{2}\left(\tau^{1 / 2} L^{2}(\Omega)\right) .
\end{aligned}
$$

Under the same hypothesis, with $\mathscr{P}(\cdot)=\Pi_{h}^{\perp}(\cdot)$, the stability results are:

$$
\begin{aligned}
& \left\{\boldsymbol{u}_{h}^{n+1}\right\}_{n=0}^{N-1} \in \ell^{\infty}\left(\boldsymbol{L}^{2}(\Omega)\right) \cap \ell^{2}\left(\boldsymbol{H}_{0}^{1}(\Omega)\right), \\
& \left\{\delta_{t} \boldsymbol{u}_{h}^{n+1}\right\}_{n=0}^{N-1} \in \ell^{2}\left(\tau^{1 / 2} \mathbf{L}^{2}(\Omega)\right), \\
& \left\{\nabla p_{h}^{n+1}\right\}_{n=0}^{N-1} \in \ell^{2}\left(\tau^{1 / 2} L^{2}(\Omega)\right) .
\end{aligned}
$$

In fact, the stability results in Corollary 4.2 are not only restricted to the backward Euler scheme but are valid for any $\mathscr{A}$-stable time integration (see Remark 4.2).

Remark 4.8. In order to satisfy the time discrete version of (38), $\boldsymbol{u}_{h}^{0}+\tilde{\boldsymbol{u}}^{0}$ must satisfy (31b). Otherwise instabilities can appear, as pointed out in [10]. An admissible choice is to take $\boldsymbol{u}_{h}^{0}$ as the Riesz projector of the initial exact solution (see [10]) and $\tilde{\boldsymbol{u}}^{0}$ equal to zero.

Remark 4.9. We refer the reader to Codina et al. [15] for an explanation about how to implement dynamic subscales.

\subsection{Stabilized formulation using quasi-static subscales}

In this section, we analyze the numerical approximation of the Stokes system using quasi-static subscales, in order to show how the stability properties are affected when the subgrid time derivative is neglected.

When using $\mathscr{P}(\cdot)=\mathscr{I}(\cdot)$, stability results can only be attained for the discrete problem using backward Euler for the time integration and assuming (1) is true. We analyze this case in Theorem 4.4.

We also prove that OSS is absolutely stable even for quasi-static subscales without any assumption over the time step size, both for the semi-discrete and fully discrete problems. Stability bounds for this case are obtained in Theorem 4.3 and Corollary 4.3.

\subsubsection{Semi-discrete problem (in space)}

When using quasi-static subscales we neglect the time derivative of the subscale, that is, $\partial_{t} \tilde{\boldsymbol{u}} \cong 0$. The problem consists of finding $\boldsymbol{u}_{h} \in L^{2}\left(0, T ; \mathscr{V}_{h}\right)$ and $p_{h} \in L^{2}\left(0, T ; \mathscr{Q}_{h}\right)$ such that:

$$
\begin{aligned}
& \left(\partial_{t} \boldsymbol{u}_{h}(t), \boldsymbol{v}_{h}\right)+v\left(\nabla \boldsymbol{u}_{h}(t), \nabla \boldsymbol{v}_{h}\right)-\left(p_{h}(t), \nabla \cdot \boldsymbol{v}_{h}\right)=\left\langle\boldsymbol{f}(t), \boldsymbol{v}_{h}\right\rangle, \\
& \left(q_{h}, \nabla \cdot \boldsymbol{u}_{h}(t)\right)-\left(\tilde{\boldsymbol{u}}(t), \nabla q_{h}\right)=0,
\end{aligned}
$$

where the subgrid component is modelled by

$$
\tau^{-1} \tilde{\boldsymbol{u}}(t)=\mathscr{P}\left(\boldsymbol{f}(t)-\nabla p_{h}(t)-\partial_{t} \boldsymbol{u}_{h}(t)\right)
$$

This is the standard methodology used for the stabilization of this mixed problem. However, as pointed out by Bochev et al. [4], this method is unstable under anisotropic space-time discretization.

It is not the aim of this section to prove the instability of these methods, we refer to Bochev et al. [4] for that. We will identify which are the main differences with respect to the dynamic subscales analyzed above. By numerical experimentation (see [4,15]), it has been shown that these small differences make the quasi-static approach unstable in the limit $\delta t \rightarrow 0$, whereas the dynamic subscales technique remains stable. The question we want to answer is: where the previous analysis fails when taking out $\partial_{t} \tilde{\boldsymbol{u}}(t)$ in (26a) and (27)?

In what follows we want to stress the effect of the choice of the subgrid projection operator $\mathscr{P}(\cdot)$. First, let us consider $\mathscr{P}(\cdot)=\mathscr{I}(\cdot)$.

In order to obtain a first set of velocity bounds, we should test (42a) with $\boldsymbol{v}_{h}=\boldsymbol{u}_{h}(t)$ and (42b) with $q_{h}=p_{h}(t)$. Finally, we would multiply (43) by $\tilde{\boldsymbol{u}}(t)$ and integrate over the whole domain. Doing that, we do not have control over the terms related to the time derivatives. At the left hand side we have:

$$
\left(\partial_{t} \boldsymbol{u}_{h}(t), \tilde{\boldsymbol{u}}(t)\right) .
$$

There is no way to control

$$
\int_{0}^{T}\left\|\partial_{t} \boldsymbol{u}_{h}(s)\right\|_{0}^{2} d s
$$


Thus, we cannot even recover velocity bounds! We could try to get first stability results over the time derivative, in order to control this term. We should take $\boldsymbol{v}_{h}=\partial_{t} \boldsymbol{u}_{h}(t)$ in (42a) and $q_{h}=p_{h}(t)$ at the time derivative of (42b). For the subgrid component, we should multiply (43) by $\partial_{t} \tilde{\boldsymbol{u}}(t)$. Now the term:

$$
\left(\boldsymbol{f}(t)-\partial_{t} \boldsymbol{u}_{h}(t), \partial_{t} \tilde{\boldsymbol{u}}(t)\right)
$$

cannot be bounded because $\left\|\partial_{t} \tilde{\boldsymbol{u}}(t)\right\|_{0}$ is not controlled.

The situation is better using OSS. Taking $\mathscr{P}(\cdot)=\Pi_{h}^{\perp}(\cdot)$, we have the following expression for the subgrid component:

$$
\tau^{-1} \tilde{\boldsymbol{u}}(t)=\Pi_{h}^{\perp}\left(\boldsymbol{f}(t)-\nabla p_{h}(t)\right)
$$

Let us introduce the following space of functions:

$$
X=\left\{\boldsymbol{f}(t) \mid \partial_{t} \boldsymbol{f}(t) \in \boldsymbol{L}^{2}\left(0, T ; \tau^{1 / 2} \mathbf{L}^{2}(\Omega)\right), \boldsymbol{f}(t) \in \boldsymbol{L}^{\infty}\left(0, T ; \boldsymbol{L}^{2}(\Omega)\right)\right\},
$$

and the norm:

$$
\|\boldsymbol{f}(t)\|_{X}^{2}=\int_{0}^{t}\left\|\tau^{1 / 2} \partial_{t} \boldsymbol{f}(s)\right\|_{0}^{2} d s+\sup _{s \in[0, T]}\|\boldsymbol{f}(s)\|_{0}^{2}
$$

used for the statement of the following theorem.

Theorem 4.3. Given a force vector sequence $\boldsymbol{f}(t) \in \boldsymbol{L}^{2}\left(0, T ; \boldsymbol{H}^{-1}(\Omega)\right) \cap \boldsymbol{L}^{2}\left(0, T ; \tau^{1 / 2} \boldsymbol{L}^{2}(\Omega)\right) \cap \tau X$ and initial condition $\boldsymbol{u}^{0} \in \boldsymbol{L}^{2}(\Omega)$, the solution $\left\{\boldsymbol{u}_{h}(t), p_{h}(t)\right\}$ of system(42) and (43) with $\mathscr{P}(\cdot)=\Pi_{h}^{\perp}(\cdot)$ satisfies:

$$
\begin{aligned}
& \boldsymbol{u}_{h}(t) \in L^{\infty}\left(0, T ; \boldsymbol{L}^{2}(\Omega)\right) \cap L^{2}\left(0, T ; \boldsymbol{H}_{0}^{1}(\Omega)\right), \\
& \partial_{t} \boldsymbol{u}_{h}(t) \in L^{2}\left(0, T ; \tau^{1 / 2} \boldsymbol{L}^{2}(\Omega)\right), \\
& \nabla p_{h}(t) \in L^{2}\left(0, T ; \tau^{1 / 2} \boldsymbol{L}^{2}(\Omega)\right) .
\end{aligned}
$$

Proof. We follow the strategy commented above. The main difference is the fact that now $\partial_{t} \boldsymbol{u}_{h}$ does not appear in (43), due to the orthogonality property. We get

$$
\left\|\boldsymbol{u}_{h}(T)\right\|_{0}^{2}+v \int_{0}^{T}\left\|\nabla \boldsymbol{u}_{h}(s)\right\|_{0}^{2} d s+\int_{0}^{T}\left\|\tau^{-1 / 2} \tilde{\boldsymbol{u}}(s)\right\|_{0}^{2} d s \leqslant \frac{C_{\Omega}}{v} \int_{0}^{T}\|\boldsymbol{f}(s)\|_{-1}^{2} d s+\int_{0}^{T}\left\|\tau^{1 / 2} \Pi_{h}^{\perp}(\boldsymbol{f}(s))\right\|_{0}^{2} d s+\left\|\boldsymbol{u}_{h}^{0}\right\|_{0}^{2} .
$$

In order to get pressure bounds, we need to control the time derivative of the finite element velocity. Using the strategy pointed out above, we get

$$
\begin{aligned}
& \int_{0}^{T}\left\|\tau^{1 / 2} \partial_{t} \boldsymbol{u}_{h}(s)\right\|_{0}^{2} d s+v\left\|\tau^{1 / 2} \nabla \boldsymbol{u}_{h}(T)\right\|_{0}^{2}+\|\tilde{\boldsymbol{u}}(T)\|_{0}^{2} \\
& \quad \leqslant \int_{0}^{T}\left\|\tau^{1 / 2} \boldsymbol{f}(s)\right\|_{0}^{2} d s+2 \int_{0}^{T} \tau\left(\boldsymbol{f}(s), \partial_{t} \tilde{\boldsymbol{u}}(s)\right) d s+v\left\|\tau^{1 / 2} \nabla \boldsymbol{u}_{h}(0)\right\|_{0}^{2}+\|\tilde{\boldsymbol{u}}(0)\|_{0}^{2} .
\end{aligned}
$$

Integrating by parts (in time) the last term and using Cauchy-Schwarz and Young's inequalities we obtain:

$$
2 \int_{0}^{T} \tau\left(\boldsymbol{f}(s), \partial_{t} \tilde{\boldsymbol{u}}(s)\right) d s \leqslant \int_{0}^{T}\left\|\tau^{3 / 2} \partial_{t} \boldsymbol{f}(s)\right\|_{0}^{2} d s+\int_{0}^{T}\left\|\tau^{-1 / 2} \tilde{\boldsymbol{u}}(s)\right\|_{0}^{2} d s+2\|\tau \boldsymbol{f}(T)\|_{0}^{2}+2\|\tau \boldsymbol{f}(0)\|_{0}^{2}+\frac{1}{2}\|\tilde{\boldsymbol{u}}(T)\|_{0}^{2}+\frac{1}{2}\|\tilde{\boldsymbol{u}}(0)\|_{0}^{2} .
$$

With these bounds, the pressure stability is obtained as in Theorem 4.2.

Remark 4.10. Let us stress the fact that the stability bounds in Theorem 4.3 can only be proved for $\mathscr{P}(\cdot)=\Pi_{h}^{\perp}(\cdot)$. Therefore, OSS remains unconditionally stable (under mild regularity properties over the body force), whereas classical residual-based stabilization techniques (with $\mathscr{P}(\cdot)=\mathscr{I}(\cdot)$ ) become unstable.

\subsubsection{Fully discrete problem}

The discretization in time of the stabilized system (42b) leads to

$$
\begin{aligned}
& \left(\delta_{t} \boldsymbol{u}_{h}^{n+1}, \boldsymbol{v}_{h}\right)+v\left(\nabla \boldsymbol{u}_{h}^{n+1}, \nabla \boldsymbol{v}_{h}\right)-\left(p_{h}^{n+1}, \nabla \cdot \boldsymbol{v}_{h}\right)=\left\langle\boldsymbol{f}^{n+1}, \boldsymbol{v}_{h}\right\rangle \\
& \left(q_{h}, \nabla \cdot \boldsymbol{u}_{h}^{n+1}\right)-\left(\tilde{\boldsymbol{u}}^{n+1}, \nabla q_{h}\right)=0 \\
& \tau^{-1} \tilde{\boldsymbol{u}}^{n+1}=\mathscr{P}\left(\boldsymbol{f}^{n+1}-\nabla p_{h}^{n+1}-\delta_{t} \boldsymbol{u}_{h}^{n+1}\right) .
\end{aligned}
$$

In this section, we show that the discrete problem for $\mathscr{P}(\cdot)=\mathscr{I}(\cdot)$ improves the stability properties of the continuous (in time) case. Using backward Euler, the discrete counterpart of the uncontrolled term (44) 


$$
\sum_{n=0}^{N-1} \delta t\left(\delta_{t} \boldsymbol{u}_{h}, \tilde{\boldsymbol{u}}\right)
$$

can be controlled by the numerical dissipation of the time integration scheme under the following assumption over the time-space discretization:

$$
\tau=\frac{C_{\tau} h^{2}}{v} \leqslant C_{\delta t} \delta t
$$

with $C_{\delta t}$ uniform with respect to the mesh size $h$ and time step size $\delta t$. Condition (50) implies that we cannot reduce the time step size without remeshing (in space). Therefore, for some anisotropic space-time discretization, we cannot prove stability results. In fact, as pointed out in [4], numerical experimentation shows that the stabilized method becomes unstable in these cases (see also [15]). It has to be remarked that for some stabilization methods the stabilization parameter is directly $\delta t$, thus making condition (50) meaningless [16]. However, for these methods stability deteriorates as $\delta t \rightarrow 0$.

This assumption over the time step size is useful together with backward Euler for the proof of stability results (see Theorem 4.4). Unfortunately, that is not enough for second order schemes. For BDF2, the numerical dissipation is not enough for controlling (49), and Crank-Nicholson does not introduce any numerical dissipation.

In the next theorems we prove stability for backward Euler, under assumption (50).

Theorem 4.4. Given a force vector sequence $\left\{\boldsymbol{f}^{n+1}\right\}_{n=0}^{N-1} \in \ell^{2}\left(\boldsymbol{H}^{-1}(\Omega)\right) \cap \ell^{2}\left(\tau^{1 / 2} \boldsymbol{L}^{2}(\Omega)\right)$, initial condition $\boldsymbol{u}^{0} \in \boldsymbol{L}^{2}(\Omega)$, a stabilization parameter satisfying (50) and a time step size such that $C_{\delta t}^{-1}<1$, the sequence $\left\{\boldsymbol{u}_{h}^{n+1}, p_{h}^{n+1}\right\}_{n=0}^{N-1}$ of solutions of system (48c) with $\mathscr{P}(\cdot)=\mathscr{I}(\cdot)$ satisfies:

$$
\begin{aligned}
& \left\{\boldsymbol{u}_{h}^{n+1}\right\}_{n=0}^{N-1} \in \ell^{\infty}\left(\boldsymbol{L}^{2}(\Omega)\right) \cap \ell^{2}\left(\boldsymbol{H}_{0}^{1}(\Omega)\right), \\
& \left\{\nabla p_{h}^{n+1}\right\}_{n=0}^{N-1} \in \ell^{2}\left(\tau^{1 / 2} \mathbf{L}^{2}(\Omega)\right) .
\end{aligned}
$$

Proof. We test (48a) with $\boldsymbol{v}_{h}=\boldsymbol{u}_{h}^{n+1}$, (48b) with $q_{h}=p_{h}^{n+1}$ and (48c) is multiplied by $\tilde{\boldsymbol{u}}^{n+1}$ and integrated over $\Omega$. Adding up the resulting equations for all the time step values, $n=0$ to $N-1$, we obtain:

$$
\begin{aligned}
& \left\|\boldsymbol{u}_{h}^{N}\right\|_{0}^{2}+\sum_{n=0}^{N}\left\|\delta \boldsymbol{u}_{h}^{n+1}\right\|_{0}^{2}+v \sum_{n=0}^{N-1} \delta t\left\|\nabla \boldsymbol{u}_{h}^{n+1}\right\|_{0}^{2}+\sum_{n=0}^{N-1} \delta t\left\|\tau^{-1 / 2} \tilde{\boldsymbol{u}}^{n+1}\right\|_{0}^{2} \\
& \leqslant \frac{C_{\Omega}}{v} \sum_{n=0}^{N-1} \delta t\left\|\boldsymbol{f}^{n+1}\right\|_{-1}^{2}+\sum_{n=0}^{N-1} \delta t\left\|\tau^{1 / 2} \boldsymbol{f}^{n+1}\right\|_{0}^{2}+\left\|\boldsymbol{u}_{h}^{0}\right\|_{0}^{2}+2 \sum_{n=0}^{N-1} \delta t\left(\delta_{t} \boldsymbol{u}_{h}^{n+1}, \tilde{\boldsymbol{u}}^{n+1}\right) .
\end{aligned}
$$

The key is how to control the last term in the right hand side. At this point, the numerical dissipation of backward Euler, $\sum_{n=0}^{N-1}\left\|\delta \boldsymbol{u}_{h}^{n+1}\right\|_{0}^{2}$ is essential. We have:

$$
2 \sum_{n=0}^{N-1} \delta t\left(\delta_{t} \boldsymbol{u}_{h}^{n+1}, \tilde{\boldsymbol{u}}^{n+1}\right) \leqslant \sum_{n=0}^{N}\left\|\delta \boldsymbol{u}_{h}^{n+1}\right\|_{0}^{2}+C_{\delta t} \sum_{n=0}^{N-1} \delta t\left\|\tau^{-1 / 2} \tilde{\boldsymbol{u}}^{n+1}\right\|_{0}^{2},
$$

where we have used (50). We can only control the last term of the previous inequality if condition $C_{\delta t} \leqslant 1$ holds.

We can recover some pressure stability, even without control over the discrete time derivative. Multiplying (48c) by $\nabla p_{h}^{n+1}$, integrating over $\Omega$ and adding for all time steps, we obtain

$$
\frac{1}{3} \sum_{n=0}^{N-1} \delta t\left\|\tau^{1 / 2} \nabla p_{h}^{n+1}\right\|_{0}^{2} C_{\delta t} \sum_{n=0}^{N-1} \delta t\left\|\delta \boldsymbol{u}_{h}^{n+1}\right\|_{0}^{2}+\sum_{n=0}^{N-1} \delta t\left\|\tau^{-1 / 2} \tilde{\boldsymbol{u}}^{n+1}\right\|_{0}^{2}+\sum_{n=0}^{N-1} \delta t\left\|\tau^{1 / 2} \boldsymbol{f}^{n+1}\right\|_{0}^{2}
$$

This result concludes the proof of the theorem.

Remark 4.11. $\Delta_{h} \boldsymbol{u}_{h}$ plays a role in this result. For high order finite elements, this term could be considered for obtaining the subgrid component (see Remark 3.3). We must add $v \Delta_{h} \boldsymbol{u} h$ to the right hand side of (43). However, using integration by parts in time, inverse estimates and the expression of the stabilization parameter, this term could also be controlled:

$$
\begin{aligned}
2 v \sum_{n=0}^{N-1} \delta t \tau\left(\Delta_{h} \boldsymbol{u}_{h}^{n+1}, \partial_{t} \tilde{\boldsymbol{u}}^{n+1}\right) \leqslant & \frac{C_{\tau}^{2} C_{h}^{2}}{4 \alpha_{1}}\left(\left\|\boldsymbol{u}_{h}^{N}\right\|_{0}^{2}+\left\|\boldsymbol{u}_{h}^{0}\right\|_{0}^{2}\right)+\alpha_{2} \sum_{n=0}^{N-1} \delta t\left\|\tau^{1 / 2} \delta_{t} \boldsymbol{u}_{h}^{n+1}\right\|_{0}^{2}+\alpha_{1}\left(\left\|\tilde{\boldsymbol{u}}^{N}\right\|_{0}^{2}+\left\|\tilde{\boldsymbol{u}}^{0}\right\|_{0}^{2}\right) \\
& +\frac{C_{\tau}^{2} C_{h}^{2}}{4 \alpha_{2}} \sum_{n=0}^{N-1} \delta t\left\|\tau^{-1 / 2} \tilde{\boldsymbol{u}}^{n+1}\right\|_{0}^{2} .
\end{aligned}
$$

Again, the stability properties of the stabilized system with $\mathscr{P}(\cdot)=\Pi_{h}^{\perp}(\cdot)$ are much better. Even though condition (50) has been used for proving stability of OSS, the new result of the previous section says that this condition is not needed in this case. We state the stability results in the following corollary. Again, the proof is analogous to the one for its semi-discrete counterpart. In this result we need the discrete version of (47), which is 


$$
X_{\delta t}=\left\{\left\{\boldsymbol{f}^{n}\right\} \mid \delta_{t} \boldsymbol{f}^{n} \in \ell^{2}\left(\tau^{1 / 2} \mathbf{L}^{2}(\Omega)\right),\left\{\boldsymbol{f}^{n}\right\} \in \ell^{\infty}\left(\boldsymbol{L}^{2}(\Omega)\right)\right\} .
$$

Corollary 4.3. Given a force vector sequence $\left\{\boldsymbol{f}^{n+1}\right\}_{n=0}^{N-1} \in \ell^{2}\left(\boldsymbol{H}^{-1}(\Omega)\right) \cap \ell^{2}\left(\tau^{1 / 2} \mathbf{L}^{2}(\Omega)\right) \cap \tau X_{\delta t}$ and initial condition $\boldsymbol{u}^{0} \in \boldsymbol{L}^{2}(\Omega)$, the sequence $\left\{\boldsymbol{u}_{h}^{n+1}, p_{h}^{n+1}\right\}_{n=0}^{N-1}$ solution of system (48c) with $\mathscr{P}(\cdot)=\Pi_{h}^{\perp}(\cdot)$ satisfies:

$$
\begin{aligned}
& \left\{\boldsymbol{u}_{h}^{n+1}\right\}_{n=0}^{N-1} \in \ell^{\infty}\left(\boldsymbol{L}^{2}(\Omega)\right) \cap \ell^{2}\left(\boldsymbol{H}_{0}^{1}(\Omega)\right), \\
& \left\{\delta_{t} \boldsymbol{u}_{h}^{n+1}\right\}_{n=0}^{N-1} \in \ell^{2}\left(\tau^{1 / 2} \mathbf{L}^{2}(\Omega)\right), \\
& \left\{\nabla p_{h}^{n+1}\right\}_{n=0}^{N-1} \in \ell^{2}\left(\tau^{1 / 2} \boldsymbol{L}^{2}(\Omega)\right) .
\end{aligned}
$$

Remark 4.12. The results in Corollary 4.3 can be easily extended to other $\mathscr{A}$-stable time integration schemes, since the numerical dissipation of the backward Euler scheme has not been exploited. On the contrary, the stability properties obtained in Theorem 4.4 do not hold for other time integration schemes since the numerical dissipation of the backward Euler scheme has been used.

\section{Error analysis for dynamic and orthogonal subscales}

In this section, we tackle the convergence analysis of the discrete Stokes problem (13) stabilized using dynamic orthogonal subscales. The convergence analysis for quasi-static orthogonal subscales can be found in [3] (for the nonlinear NavierStokes equations). It has to be pointed out that convergence using ASGS is considerably more involved and, as far as we are aware, it has been undertaken only using space-time finite elements (in [18], for example, also in the context of the NavierStokes equations). The analysis of other stabilized formulations that are not residual-based can be found in [10], where condition (50) is circumvented by using an appropriate projection of the initial condition (recall that we have proved stability for quasi-static orthogonal subscales without condition (50)).

Invoking the fact that the subscale is orthogonal to the finite element space, system (41) can be further simplified. This scheme reads as follows: given $\boldsymbol{u}_{h}^{n}$, find $\boldsymbol{u}_{h}^{n+1} \in \mathscr{V}_{h}$ and $p_{h}^{n+1} \in \mathscr{Q}_{h}$ such that:

$$
\begin{aligned}
& \left(\delta_{t} \boldsymbol{u}_{h}^{n+1}, \boldsymbol{v}_{h}\right)+v\left(\nabla \boldsymbol{u}_{h}^{n+1}, \nabla \boldsymbol{v}_{h}\right)-\left(p_{h}^{n+1}, \nabla \cdot \boldsymbol{v}_{h}\right)=\left\langle\boldsymbol{f}^{n+1}, \boldsymbol{v}_{h}\right\rangle, \\
& \left(q_{h}, \nabla \cdot \boldsymbol{u}_{h}^{n+1}\right)-\left(\tilde{\boldsymbol{u}}^{n+1}, \nabla q_{h}\right)=0,
\end{aligned}
$$

initialized with $\boldsymbol{u}_{h}^{0}=\Pi_{R}\left(\boldsymbol{u}^{0}\right)$ (where $\Pi_{R}$ is the Riesz projector is used in [10]). In the previous section strong stability bounds have been obtained for this scheme. In this section optimal a priori error estimates are proved. Instead of (41), a non-residual version of the subgrid component is analyzed,

$$
\delta_{t} \tilde{\boldsymbol{u}}^{n+1}+\tau^{-1} \tilde{\boldsymbol{u}}^{n+1}=-\Pi_{h}^{\perp}\left(\nabla p_{h}^{n+1}\right) .
$$

The subgrid component is initialized with $\tilde{\boldsymbol{u}}^{0}=0$. At the right hand side of the subgrid equation only the pressure term is considered, instead of the whole residual. Even in this case orthogonal subscales keep optimality, as proved below. In fact, that is possible because of the introduction of an orthogonal projection. Similar results can be obtained using the residual form.

Let us introduce the following error functions:

$$
\begin{aligned}
& \boldsymbol{e}_{d}^{n+1}=\boldsymbol{u}\left(t^{n+1}\right)-\boldsymbol{u}_{h}^{n+1}, \\
& r_{d}^{n+1}=p\left(t^{n+1}\right)-p_{h}^{n+1}, \\
& \tilde{\boldsymbol{e}}_{d}^{n+1}=\tilde{\boldsymbol{u}}_{e}^{n+1}-\tilde{\boldsymbol{u}}^{n+1}
\end{aligned}
$$

where $\tilde{\boldsymbol{u}}_{e}^{n+1}$ is defined below. Subtracting the discrete system (51) to the continuous problem (10) evaluated at $t^{n+1}$ we get the error system:

$$
\begin{aligned}
& \left(\delta_{t} \boldsymbol{e}_{d}^{n+1}, \boldsymbol{v}_{h}\right)+v\left(\nabla \boldsymbol{e}_{d}^{n+1}, \nabla \boldsymbol{v}_{h}\right)-\left(r_{d}^{n+1}, \nabla \cdot \boldsymbol{v}_{h}\right)=\left(\delta_{t} \boldsymbol{u}\left(t^{n+1}\right)-\partial_{t} \boldsymbol{u}\left(t^{n+1}\right), \boldsymbol{v}_{h}\right), \\
& \left(q_{h}, \nabla \cdot \boldsymbol{e}_{d}^{n+1}\right)-\left(\tilde{\boldsymbol{e}}_{d}^{n+1}, \nabla q_{h}\right)=-\left(\tilde{\boldsymbol{u}}_{e}^{n+1}, \nabla q_{h}\right),
\end{aligned}
$$

where the exact subgrid velocity is defined by

$$
\delta_{t} \tilde{\boldsymbol{u}}_{e}^{n+1}+\tau^{-1} \tilde{\boldsymbol{u}}_{e}^{n+1}=-\Pi_{h}^{\perp}\left(\nabla p\left(t^{n+1}\right)\right) .
$$

and initialized with $\tilde{\boldsymbol{u}}_{e}^{0}=\mathbf{0}$. Therefore, the subgrid error is governed by

$$
\delta_{t} \tilde{\boldsymbol{e}}_{d}^{n+1}+\tau^{-1} \tilde{\boldsymbol{e}}_{d}^{n+1}=-\Pi_{h}^{\perp}\left(\nabla r_{d}^{n+1}\right) .
$$

In order to state the convergence results of the following theorem in a compact way, let us introduce the following (squared) space interpolation error function: 


$$
\begin{aligned}
\mathscr{I}_{h}= & \sum_{n=0}^{N-1} \delta t\left\{\left\|\tau^{-1 / 2} \boldsymbol{i}^{n+1}\right\|_{0}^{2}+v\left\|\nabla \boldsymbol{i}^{n+1}\right\|_{0}^{2}+v^{-1}\left\|s^{n+1}\right\|_{0}^{2}+\left\|\tau^{1 / 2} \nabla s^{n+1}\right\|_{0}^{2}+\left\|\tau^{1 / 2} \delta_{t} \boldsymbol{i}^{n+1}\right\|_{0}^{2}\right. \\
& \left.+v\left\|\tau \nabla \delta_{t} \boldsymbol{i}^{n+1}\right\|_{0}^{2}+\left\|\tau^{1 / 2} \Pi_{h}^{\perp}\left(\nabla p\left(t^{n+1}\right)\right)\right\|_{0}^{2}\right\}+\left\|\boldsymbol{i}^{0}\right\|_{0}^{2}+v\left\|\tau^{1 / 2} \nabla \boldsymbol{i}^{0}\right\|_{0}^{2}
\end{aligned}
$$

with

$$
\begin{aligned}
\boldsymbol{i}^{n+1} & =\boldsymbol{u}\left(t^{n+1}\right)-\Pi_{h}\left(\boldsymbol{u}\left(t^{n+1}\right)\right) \in \mathscr{V}_{h}^{\perp}, \\
s^{n+1} & =p\left(t^{n+1}\right)-\Pi_{h}\left(p\left(t^{n+1}\right)\right) \in \mathscr{2}_{h}^{\perp} .
\end{aligned}
$$

Let us also define the (squared) time interpolation error function:

$$
\mathscr{I}_{\delta t}=\sum_{n=0}^{N-1} \delta t v^{-1} C_{\Omega}^{2}\left\|\delta_{t} \boldsymbol{u}\left(t^{n+1}\right)-\partial_{t} \boldsymbol{u}\left(t^{n+1}\right)\right\|_{-1}^{2}+\sum_{n=0}^{N-1} \delta t\left\|\tau^{1 / 2}\left(\delta_{t} \boldsymbol{u}\left(t^{n+1}\right)-\partial_{t} \boldsymbol{u}\left(t^{n+1}\right)\right)\right\|_{0}^{2} .
$$

In the following lemma the order of accuracy with respect to the space and time discretization is analyzed. Let us introduce the following norm:

$$
\|f\|_{Y}^{2}=\|f\|_{-1}^{2}+\left\|\tau^{1 / 2} f\right\|_{0}^{2}+\|\tau \nabla f\|_{0}^{2} .
$$

Lemma 5.1. The space and time interpolation error functions defined in (56) and (57) satisfy:

$$
\mathscr{I}_{h}+\mathscr{I}_{\delta t} \leqslant C \sum_{n=0}^{N-1} \delta t \tau^{-1} h^{2(k+1)}\left\{\left\|\boldsymbol{u}\left(t^{n+1}\right)\right\|_{k+1}^{2}+v^{-2}\left\|p\left(t^{n+1}\right)\right\|_{k}^{2}+v^{-2}\left\|\partial_{t} \boldsymbol{u}\left(t^{n+1}\right)\right\|_{k-1}^{2}\right\}+C \delta t \int_{0}^{T}\left\|\partial_{t t} \boldsymbol{u}(s)\right\|_{Y}^{2} d s+C h^{2 k}\left\|\boldsymbol{u}^{0}\right\|_{k}^{2},
$$

$k$ being the degree of interpolation of the finite element approximation, for equal order velocity-pressure approximations. Furthermore, if the inequality $\tau \leqslant C_{\delta t} \delta t$ holds with $C_{\delta t}$ uniform with respect to $h$ and $\delta t$, these error functions satisfy:

$$
\mathscr{I}_{h}+\mathscr{I}_{\delta t} \leqslant C \sum_{n=0}^{N-1} \delta t \tau^{-1} h^{2(k+1)}\left\{\left\|\boldsymbol{u}\left(t^{n+1}\right)\right\|_{k+1}^{2}+v^{-2}\left\|p\left(t^{n+1}\right)\right\|_{k}^{2}\right\}+C \delta t \int_{0}^{T}\left\|\partial_{t t} \boldsymbol{u}(s)\right\|_{Y}^{2} d s+C h^{2 k}\left\|\boldsymbol{u}^{0}\right\|_{k}^{2} .
$$

Proof. Using classical interpolation results and assuming regularity over the continuous velocity we can optimally bound some terms of the space error function:

$$
\begin{aligned}
& \sum_{n=0}^{N-1} \delta t\left\{\left\|\tau^{-1 / 2} \boldsymbol{i}^{n+1}\right\|_{0}^{2}+v\left\|\nabla \boldsymbol{i}^{n+1}\right\|_{0}^{2}+v^{-1}\left\|s^{n+1}\right\|_{0}^{2}+\left\|\tau^{1 / 2} \nabla s^{n+1}\right\|_{0}^{2}+\left\|\tau^{1 / 2} \Pi_{h}^{\perp}\left(\nabla p\left(t^{n+1}\right)\right)\right\|_{0}^{2}\right\}+\left\|\boldsymbol{i}^{0}\right\|_{0}^{2}+v\left\|\tau^{1 / 2} \nabla \boldsymbol{i}^{0}\right\|_{0}^{2} \\
& \quad \leqslant C \sum_{n=0}^{N-1} \delta t \tau^{-1} h^{2(k+1)}\left\{\left\|\boldsymbol{u}\left(t^{n+1}\right)\right\|_{k+1}^{2}+v^{-2}\left\|p\left(t^{n+1}\right)\right\|_{k}^{2}\right\}+C h^{2 k}\left\|\boldsymbol{u}^{0}\right\|_{k}^{2} .
\end{aligned}
$$

The terms related to the time derivative can be easily handled under the condition $\tau \leqslant C \delta t$. But this assumption can be replaced by some extra regularity assumptions. First, let us bound the discrete time derivatives in terms of continuous derivatives using the Taylor's expansion with the residual in the integral form:

$$
\begin{aligned}
& \sum_{n=0}^{N-1} \delta t\left\|\tau^{1 / 2} \delta_{t} \boldsymbol{i}^{n+1}\right\|_{0}^{2} \leqslant \sum_{n=0}^{N-1} \delta t\left\|\tau^{1 / 2} \partial_{t} \boldsymbol{i}^{n+1}\right\|_{0}^{2}+C \delta t^{2} \int_{0}^{T}\left\|\tau^{1 / 2} \partial_{t t} \boldsymbol{u}(t)(s)\right\|_{0}^{2} d s, \\
& \sum_{n=0}^{N-1} \delta t v\left\|\tau \nabla \delta_{t} \boldsymbol{i}^{n+1}\right\|_{0}^{2} \leqslant \sum_{n=0}^{N-1} \delta t v\left\|\tau \nabla \partial_{t} \boldsymbol{i}^{n+1}\right\|_{0}^{2}+C \delta t^{2} v \int_{0}^{T}\left\|\tau \nabla \partial_{t t} \boldsymbol{u}(t)(s)\right\|_{0}^{2} d s .
\end{aligned}
$$

Again, from classical interpolation results, we can get:

$$
\tau^{2} v\left\|\nabla \partial_{t} \boldsymbol{i}^{n+1}\right\|_{0}^{2}+\tau\left\|\partial_{t} \boldsymbol{i}^{n+1}\right\|_{0}^{2} \leqslant C v^{-2} \tau^{-1} h^{2(k+1)}\left\|\partial_{t} \boldsymbol{u}\left(t^{n+1}\right)\right\|_{k-1}^{2} .
$$

The last term that must be considered is the one related to the time integration, that can be bounded using again Taylor's expansions:

$$
\begin{aligned}
& \sum_{n=0}^{N-1} \delta t v^{-1} C_{\Omega}^{2}\left\|\delta_{t} \boldsymbol{u}\left(t^{n+1}\right)-\partial_{t} \boldsymbol{u}\left(t^{n+1}\right)\right\|_{-1}^{2}+\sum_{n=0}^{N-1} \delta t\left\|\tau^{1 / 2}\left(\delta_{t} \boldsymbol{u}\left(t^{n+1}\right)-\partial_{t} \boldsymbol{u}\left(t^{n+1}\right)\right)\right\|_{0}^{2} \\
& \quad \leqslant C \delta t^{2} \int_{0}^{T}\left(v^{-1} C_{\Omega}^{2}\left\|\partial_{t t} \boldsymbol{u}(t)(s)\right\|_{-1}^{2}+\left\|\tau^{1 / 2} \partial_{t t} \boldsymbol{u}(t)(s)\right\|_{0}^{2}\right) d s .
\end{aligned}
$$

The lemma is easily proved combining the previous inequalities. 
Remark 5.1. From the previous lemma we can infer that condition (50) allows to assume less regularity on the time derivative of the continuous velocity.

In the following theorem some convergence results have been obtained in terms of the error functions analyzed above. The proof of the finite element error contains a novel approach that involves error bounds for the subgrid component.

Theorem 5.1. The error functions $\left\{\boldsymbol{e}_{d}^{n+1}, r_{d}^{n+1}, \tilde{\boldsymbol{e}}_{d}^{n+1}\right\}$ determined by system (53) satisfy the following inequalities:

$$
\begin{aligned}
& \left\|\boldsymbol{e}_{d}^{N}\right\|_{0}^{2}+\sum_{n=0}^{N-1} \delta t v\left\|\nabla \boldsymbol{e}_{d}^{n+1}\right\|_{0}^{2}+\left\|\tilde{\boldsymbol{e}}_{d}^{N}\right\|_{0}^{2}+\sum_{n=0}^{N-1} \delta t\left\|\tau^{-1 / 2} \tilde{\boldsymbol{e}}_{d}^{n+1}\right\|_{0}^{2}+\sum_{n=0}^{N-1} \delta t\left\|\tau^{1 / 2} \delta_{t} \boldsymbol{e}_{d}^{n+1}\right\|_{0}^{2}+v\left\|\tau^{1 / 2} \nabla \boldsymbol{e}_{d}^{N}\right\|_{0}^{2}+\sum_{n=0}^{N-1} \delta t\left\|\tau^{1 / 2} \delta_{t} \tilde{\boldsymbol{e}}_{d}^{n+1}\right\|_{0}^{2} \\
& \quad \leqslant C\left(\mathscr{I}_{h}+\mathscr{I}_{\delta t}\right),
\end{aligned}
$$

and

$$
\left\|\tau^{1 / 2} \nabla r_{d}^{n+1}\right\|_{0}^{2} \leqslant C\left(C_{h}\right)\left(\mathscr{I}_{h}+\mathscr{I}_{\delta t}\right),
$$

where $C\left(C_{h}\right)$ is a positive constant depending on $C_{h}$ (defined in (33)).

Proof. Let us start the proof of the theorem testing (53a) with the finite element function $\boldsymbol{v}_{h}=\Pi_{h}\left(\boldsymbol{u}\left(t^{n+1}\right)\right)-\boldsymbol{u}_{h}^{n+1}=\boldsymbol{e}_{d}^{n+1}-\boldsymbol{i}^{n+1}$ for $n=0, \ldots, N-1$. Adding up the resulting equations for all time steps, we finally get:

$$
\begin{aligned}
\frac{1}{2}\left\|\boldsymbol{e}_{d}^{N}\right\|_{0}^{2}+\sum_{n=0}^{N-1} \delta t v\left\|\nabla \boldsymbol{e}_{d}^{n+1}\right\|_{0}^{2}-\delta t\left(r_{d}^{n+1}, \nabla \cdot \boldsymbol{e}_{d}^{n+1}\right) \leqslant & \sum_{n=0}^{N-1} \delta t\left\{\left(\delta_{t} \boldsymbol{e}_{d}^{n+1}, \boldsymbol{i}^{n+1}\right)+v\left(\nabla \boldsymbol{e}_{d}^{n+1}, \nabla \boldsymbol{i}^{n+1}\right)+\left(\nabla r_{d}^{n+1}, \boldsymbol{i}^{n+1}\right)\right. \\
& \left.+\left(\delta_{t} \boldsymbol{u}\left(t^{n+1}\right)-\partial_{t} \boldsymbol{u}\left(t^{n+1}\right), \boldsymbol{e}_{d}^{n+1}-\boldsymbol{i}^{n+1}\right)\right\}+\frac{1}{2}\left\|\boldsymbol{i}^{0}\right\|_{0}^{2} .
\end{aligned}
$$

The pressure error term at the left hand side can be handled by using (53b) tested with $q_{h}=\Pi_{h}(p)-p_{h}=r_{d}^{n+1}-s^{n+1}$ :

$$
\left(r_{d}^{n+1}, \nabla \cdot \boldsymbol{e}_{d}^{n+1}\right)-\left(\tilde{\boldsymbol{e}}_{d}^{n+1}, \nabla r_{d}^{n+1}\right)=\left(s^{n+1}, \nabla \cdot \boldsymbol{e}_{d}^{n+1}\right)-\left(\tilde{\boldsymbol{e}}_{d}^{n+1}, \nabla s^{n+1}\right)-\left(\tilde{\boldsymbol{u}}_{e}^{n+1}, \nabla\left(r_{d}^{n+1}-s^{n+1}\right)\right) .
$$

In order to bound the subgrid term let us multiply the subgrid error equation (55) by $\tilde{\boldsymbol{e}}_{d}^{n+1}$, integrate the result over $\Omega$ and add up for $n=0, \ldots, N-1$. We finally get:

$$
\frac{1}{2}\left\|\tilde{\boldsymbol{e}}_{d}^{N}\right\|_{0}^{2}+\sum_{n=0}^{N-1} \delta t\left\|\tau^{-1 / 2} \tilde{\boldsymbol{e}}_{d}^{n+1}\right\|_{0}^{2} \leqslant-\sum_{n=0}^{N-1} \delta t\left(\tilde{\boldsymbol{e}}_{d}^{n+1}, \nabla r_{d}^{n+1}\right)+\frac{1}{2}\left\|\boldsymbol{i}^{0}\right\|_{0}^{2} .
$$

Let us point out the fact that $\tilde{\boldsymbol{e}}_{d}^{0}=-\boldsymbol{i}^{0}$. Adding (63) and (65) and using expression (64), we get the following inequality:

$$
\begin{aligned}
& \frac{1}{2}\left\|\boldsymbol{e}_{d}^{N}\right\|_{0}^{2}+\sum_{n=0}^{N-1} \delta t v\left\|\nabla \boldsymbol{e}_{d}^{n+1}\right\|_{0}^{2}+\frac{1}{2}\left\|\tilde{\boldsymbol{e}}_{d}^{N}\right\|_{0}^{2}+\sum_{n=0}^{N-1} \delta t\left\|\tau^{-1 / 2} \tilde{\boldsymbol{e}}_{d}^{n+1}\right\|_{0}^{2} \\
& \leqslant \sum_{n=0}^{N-1} \delta t\left\{\left(\delta_{t} \boldsymbol{e}_{d}^{n+1}, \boldsymbol{i}^{n+1}\right)+v\left(\nabla \boldsymbol{e}_{d}^{n+1}, \nabla \boldsymbol{i}^{n+1}\right)+\left(\nabla r_{d}^{n+1}, \boldsymbol{i}^{n+1}\right)+\left(s^{n+1}, \nabla \cdot \boldsymbol{e}_{d}^{n+1}\right)-\left(\tilde{\boldsymbol{e}}_{d}^{n+1}, \nabla s^{n+1}\right)\right. \\
&\left.-\left(\tilde{\boldsymbol{u}}_{e}^{n+1}, \nabla\left(r_{d}^{n+1}-s^{n+1}\right)\right)+\left(\delta_{t} \boldsymbol{u}\left(t^{n+1}\right)-\partial_{t} \boldsymbol{u}\left(t^{n+1}\right), \boldsymbol{e}_{d}^{n+1}-\boldsymbol{i}^{n+1}\right)\right\}+\left\|\boldsymbol{i}^{0}\right\|_{0}^{2}:=\mathscr{R} \mathscr{H} \mathscr{S}_{1} .
\end{aligned}
$$

Let us obtain an appropriate bound for $\mathscr{R} \mathscr{H} \mathscr{S}_{1}$. Using Cauchy-Schwarz and Young's inequalities for every term of the right hand side, we arrive to:

$$
\begin{aligned}
\mathscr{R} \mathscr{H}_{1} \mathscr{S}_{1} \leqslant & \frac{\alpha}{2} \sum_{n=0}^{N-1} \delta t\left\{\left\|\tau^{1 / 2} \delta_{t} \boldsymbol{e}_{d}^{n+1}\right\|_{0}^{2}+3 v\left\|\nabla \boldsymbol{e}_{d}^{n+1}\right\|_{0}^{2}+2\left\|\tau^{1 / 2} \Pi_{h}^{\perp}\left(\nabla r_{d}^{n+1}\right)\right\|_{0}^{2}+\left\|\tau^{-1 / 2} \tilde{\boldsymbol{e}}_{d}^{n+1}\right\|_{0}^{2}\right\}+\frac{\alpha+1}{2 \alpha} \\
& \times \sum_{n=0}^{N-1} \delta t\left\{2\left\|\tau^{-1 / 2} \boldsymbol{i}^{n+1}\right\|_{0}^{2}+v\left\|\nabla \boldsymbol{i}^{n+1}\right\|_{0}^{2}+v^{-1}\left\|s^{n+1}\right\|_{0}^{2}+\left\|\tau^{1 / 2} \nabla s^{n+1}\right\|_{0}^{2}+\left\|\tau^{-1 / 2} \tilde{\boldsymbol{u}}_{e}^{n+1}\right\|_{0}^{2}\right. \\
& \left.+v^{-1} C_{\Omega}^{2}\left\|\delta_{t} \boldsymbol{u}\left(t^{n+1}\right)-\partial_{t} \boldsymbol{u}\left(t^{n+1}\right)\right\|_{-1}^{2}\right\}+\left\|\boldsymbol{i}^{0}\right\|_{0}^{2},
\end{aligned}
$$

where $\alpha$ is a positive scalar value that arises from Young's inequality. This $\alpha$ will be chosen below in order to absorb some terms by the left hand side. Let us point out that (67) involves norms of $\delta_{t} \boldsymbol{e}_{d}^{n+1}$. In order to bound these norms we test (53a) with $\boldsymbol{v}_{h}=\tau \Pi_{h}\left(\delta_{t} \boldsymbol{e}_{d}^{n+1}\right)=\tau \delta_{t}\left(\boldsymbol{e}_{d}^{n+1}-\boldsymbol{i}^{n+1}\right)$ for $n=0, \ldots, N-1$. Adding up for all time steps and carrying out algebraic manipulations, we get:

$$
\begin{aligned}
& \sum_{n=0}^{N-1} \delta t\left\|\tau^{1 / 2} \delta_{t} \boldsymbol{e}_{d}^{n+1}\right\|_{0}^{2}+\frac{v}{2}\left\|\tau^{1 / 2} \nabla \boldsymbol{e}_{d}^{N}\right\|_{0}^{2}-\sum_{n=0}^{N-1} \delta t\left(\tau r_{d}^{n+1}, \nabla \cdot \delta_{t} \boldsymbol{e}_{d}^{n+1}\right) \\
& \quad \leqslant \sum_{n=0}^{N-1} \delta t\left\{\left(\tau \delta_{t} \boldsymbol{e}_{d}^{n+1}, \delta_{t} \boldsymbol{i}^{n+1}\right)+v\left(\tau \nabla \boldsymbol{e}_{d}^{n+1}, \nabla \delta_{t} \boldsymbol{i}^{n+1}\right)+\left(\tau\left(\delta_{t} \boldsymbol{u}\left(t^{n+1}\right)-\partial_{t} \boldsymbol{u}\left(t^{n+1}\right)\right), \delta_{t} \boldsymbol{e}_{d}^{n+1}-\delta_{t} \boldsymbol{i}^{n+1}\right)+\left(\tau \nabla r_{d}^{n+1}, \delta_{t} \boldsymbol{i}^{n+1}\right)\right\} \\
& \quad+\frac{v}{2}\left\|\tau^{1 / 2} \nabla \boldsymbol{i}^{0}\right\|_{0}^{2}
\end{aligned}
$$


Let us apply the operator $\delta_{t}$ over (53b) and test the resulting equation with $q_{h}=\tau\left(r_{d}^{n+1}-s^{n+1}\right)$. It leads to the following equality:

$$
\left(\tau r_{d}^{n+1}, \nabla \cdot \delta_{t} \boldsymbol{e}_{d}^{n+1}\right)-\left(\delta_{t} \tilde{\boldsymbol{e}}_{d}^{n+1}, \tau \nabla r_{d}^{n+1}\right)=\left(\tau s^{n+1}, \nabla \cdot \delta_{t} \boldsymbol{e}_{d}^{n+1}\right)-\left(\delta_{t} \tilde{\boldsymbol{e}}_{d}^{n+1}, \tau \nabla s^{n+1}\right)+\left(\delta_{t} \tilde{\boldsymbol{u}}_{e}^{n+1}, \tau \nabla\left(r_{d}^{n+1}-s^{n+1}\right)\right) .
$$

Finally, let us multiply (55) by $\tau \delta_{t} \tilde{\boldsymbol{e}}_{d}^{n+1}$ for $n=0, \ldots, N-1$. We integrate the result over $\Omega$ and add up for all time steps. We obtain:

$$
\sum_{n=0}^{N-1} \delta t\left\|\tau^{1 / 2} \delta_{t} \tilde{\boldsymbol{e}}_{d}^{n+1}\right\|_{0}^{2}+\frac{1}{2}\left\|\tilde{\boldsymbol{e}}_{d}^{N}\right\|_{0}^{2} \leqslant-\sum_{n=0}^{N-1} \delta t\left(\delta_{t} \tilde{\boldsymbol{e}}_{d}^{n+1}, \tau \nabla r_{d}^{n+1}\right)+\frac{1}{2}\left\|\boldsymbol{i}^{0}\right\|_{0}^{2} .
$$

We add (68) and (70) and use (69) in order to get bounds for $\delta_{t} \tilde{\boldsymbol{e}}_{d}^{n+1}$ :

$$
\begin{aligned}
& \sum_{n=0}^{N-1} \delta t\left\|\tau^{1 / 2} \delta_{t} \boldsymbol{e}_{d}^{n+1}\right\|_{0}^{2}+\frac{v}{2}\left\|\tau^{1 / 2} \nabla \boldsymbol{e}_{d}^{N}\right\|_{0}^{2}+\sum_{n=0}^{N-1} \delta t\left\|\tau^{1 / 2} \delta_{t} \tilde{\boldsymbol{e}}_{d}^{n+1}\right\|_{0}^{2} \\
& \quad+\frac{1}{2}\left\|\tilde{\boldsymbol{e}}_{d}^{N}\right\|_{0}^{2} \leqslant \sum_{n=0}^{N-1} \delta t\left\{\left(\tau \delta_{t} \boldsymbol{e}_{d}^{n+1}, \delta_{t} \boldsymbol{i}^{n+1}\right)+v\left(\tau \nabla \boldsymbol{e}_{d}^{n+1}, \nabla \delta_{t} \boldsymbol{i}^{n+1}\right)+\left(\tau s^{n+1}, \nabla \cdot \delta_{t} \boldsymbol{e}_{d}^{n+1}\right)-\left(\delta_{t} \tilde{\boldsymbol{e}}_{d}^{n+1}, \tau \nabla s^{n+1}\right)\right. \\
& \left.\quad+\left(\delta_{t} \tilde{\boldsymbol{u}}_{e}^{n+1}, \tau \nabla\left(r_{d}^{n+1}-s^{n+1}\right)\right)+\left(\tau \nabla r_{d}^{n+1}, \delta_{t} \boldsymbol{i}^{n+1}\right)+\left(\tau\left(\delta_{t} \boldsymbol{u}\left(t^{n+1}\right)-\partial_{t} \boldsymbol{u}\left(t^{n+1}\right)\right), \delta_{t} \boldsymbol{e}_{d}^{n+1}-\delta_{t} \boldsymbol{i}^{n+1}\right)\right\} \\
& \quad+\frac{v}{2}\left\|\tau^{1 / 2} \nabla \boldsymbol{i}^{0}\right\|_{0}^{2}+\frac{1}{2}\left\|\boldsymbol{i}^{0}\right\|_{0}^{2}:=\mathscr{R} \mathscr{H} \mathscr{S}_{2}
\end{aligned}
$$

$\mathscr{R} \mathscr{H} \mathscr{S}_{2}$ can also be bounded using Cauchy-Schwarz and Young's inequalities:

$$
\begin{aligned}
\mathscr{R} \mathscr{H} \mathscr{S}_{2} \leqslant & \frac{\beta}{2} \sum_{n=0}^{N-1} \delta t\left\{3\left\|\tau^{1 / 2} \delta_{t} \boldsymbol{e}_{d}^{n+1}\right\|_{0}^{2}+v\left\|\nabla \boldsymbol{e}_{d}^{n+1}\right\|_{0}^{2}+2\left\|\tau^{1 / 2} \Pi_{h}^{\perp}\left(\nabla r_{d}^{n+1}\right)\right\|_{0}^{2}+\left\|\tau^{1 / 2} \delta_{t} \tilde{\boldsymbol{e}}_{d}^{n+1}\right\|_{0}^{2}\right\}+\frac{\beta+1}{2 \beta} \\
& \times \sum_{n=0}^{N-1} \delta t\left\{2\left\|\tau^{1 / 2} \delta_{t} \boldsymbol{i}^{n+1}\right\|_{0}^{2}+v\left\|\tau \nabla \delta_{t} \boldsymbol{i}^{n+1}\right\|_{0}^{2}+\left\|\tau^{1 / 2} \nabla s^{n+1}\right\|_{0}^{2}+\left\|\tau^{1 / 2} \delta_{t} \tilde{\boldsymbol{u}}_{e}^{n+1}\right\|_{0}^{2}+\left\|\delta_{t} \boldsymbol{u}\left(t^{n+1}\right)-\partial_{t} \boldsymbol{u}\left(t^{n+1}\right)\right\|_{0}^{2}\right\} \\
& +\frac{v}{2}\left\|\tau^{1 / 2} \nabla \boldsymbol{i}^{0}\right\|_{0}^{2}+\frac{1}{2}\left\|\boldsymbol{i}^{0}\right\|_{0}^{2} .
\end{aligned}
$$

Again, $\beta$ is a positive scalar value that will be selected in order to absorb some terms by the left hand side. The pressure error terms in (67) and (72) must be absorbed by left hand side terms. In fact, only the orthogonal projection $\Pi_{h}^{\perp}\left(\nabla r_{d}^{n+1}\right)$ must be controlled. From (55), we can easily get:

$$
\left\|\tau^{1 / 2} \Pi_{h}^{\perp}\left(\nabla r_{d}^{n+1}\right)\right\|_{0}^{2} \leqslant 2\left\{\left\|\tau^{-1 / 2} \tilde{\boldsymbol{e}}_{d}^{n+1}\right\|_{0}^{2}+\left\|\tau^{1 / 2} \delta_{t} \tilde{\boldsymbol{e}}_{d}^{n+1}\right\|_{0}^{2}\right\}
$$

The exact subgrid component $\tilde{\boldsymbol{u}}_{e}^{n+1}$ can be bounded from (54). We multiply this equation by $\tilde{\boldsymbol{u}}_{e}^{n+1}$, leading to:

$$
\sum_{n=0}^{N-1} \delta t\left\|\tau^{-1 / 2} \tilde{\boldsymbol{u}}_{e}^{n+1}\right\|_{0}^{2} \leqslant \sum_{n=0}^{N-1} \delta t\left\|\tau^{1 / 2} \Pi_{h}^{\perp}\left(\nabla p\left(t^{n+1}\right)\right)\right\|_{0}^{2} .
$$

On the other hand, multiplying (52) by $\tau \delta_{t} \tilde{\boldsymbol{u}}_{e}^{n+1}$, we get:

$$
\sum_{n=0}^{N-1} \delta t\left\|\tau^{1 / 2} \delta_{t} \tilde{\boldsymbol{u}}_{e}^{n+1}\right\|_{0}^{2} \leqslant \sum_{n=0}^{N-1} \delta t\left\|\tau^{1 / 2} \Pi_{h}^{\perp}\left(\nabla p\left(t^{n+1}\right)\right)\right\|_{0}^{2}
$$

Let us combine (66) and (71) together with the right hand side bounds (67) and (72) and inequalities (73)-(75). We consider $\alpha$ and $\beta$ small enough in order to absorb some terms by the left hand side. The rest of terms belong to the error functions $\mathscr{I}_{h}$ and $\mathscr{I}_{\delta t}$ defined in (56) and (57), respectively. Thus, the first part of the theorem is proved.

The pressure error requires a further step. We have got control over the orthogonal projection of $\nabla r_{d}^{n+1}$ in (73). The finite element component can be bounded taking $\boldsymbol{v}_{h}=\tau \Pi_{h}\left(\nabla r_{d}^{n+1}\right)$ in (53a) and using inverse estimate (33):

$$
\left\|\tau^{1 / 2} \Pi_{h}\left(\nabla r_{d}^{n+1}\right)\right\|_{0}^{2} \leqslant 3\left\{C_{h}^{2} v\left\|\nabla \boldsymbol{e}_{d}^{n+1}\right\|_{0}^{2}+\left\|\tau^{1 / 2} \delta_{t} \boldsymbol{e}_{d}^{n+1}\right\|_{0}^{2}+\left\|\tau^{1 / 2}\left(\delta_{t} \boldsymbol{u}\left(t^{n+1}\right)-\partial_{t} \boldsymbol{u}\left(t^{n+1}\right)\right)\right\|_{0}^{2}\right\}
$$

\section{Summary and conclusions}

In this work, we have analyzed the stability properties of some numerical techniques for the approximation of the transient Stokes system. Let us summarize the most salient results of the paper:

1. Theorem 4.1 and Corollary 4.1. The use of div-stable elements allows us to obtain appropriate stability over the pressure for the stationary case. Unfortunately, for the transient problem the regularity of the pressure is not so clear. Under a discrete inf-sup condition, the time regularity of the pressure is not evident. Under strong regularity assumptions over the data (initial velocity $\boldsymbol{u}^{0} \in \boldsymbol{H}^{1}(\Omega)$ ) strong pressure bounds can be proved. On the contrary, under minimum regularity assumptions the stability bounds over the pressure are very weak. 
2. Theorem 4.4. For classical stabilized methods (like ASGS, Galerkin/least-squares or streamline upwind/Petrov-Galerkin) applied to the transient problem, where the subgrid component is not tracked in time (quasi-static subscales), pressure stability is only attained when using the backward Euler scheme under the following restriction over the time step size:

$$
h^{2} \leqslant C \delta t
$$

with $C$ uniform with respect to $\delta t$ and $h$. In fact, if this condition is violated (anisotropic space-time discretizations), these stabilized methods become unstable. For BDF2 and Crank-Nicholson schemes, stability cannot be proved even under this assumption over the time step value.

3. Theorem 4.2 and Corollary 4.2. On the contrary, considering the tracking of the subscales in time (dynamic subscales) the situation is much better. This approach exhibits improved stability properties, allowing to get appropriate stability bounds over the pressure without any assumption over the time step size.

4. Theorem 4.3 and Corollary 4.3. Using OSS we can recover stability without any approximation over the time step size even with quasi-static subscales. Only mild regularity assumptions over the force term are needed.

5. Theorem 5.1. For the fully discrete transient Stokes problem stabilized with dynamic and orthogonal subscales we have also proved optimal convergence results. Two different cases have been considered: under an assumption over the time step size and without any assumption over $\delta t$ and slightly stronger regularity requirements.

We can conclude that it is worth to track the subscales in time in a variational multiscale approach to the Stokes problem. Dynamic subscales are naturally motivated from the multiscale concept and lead in a natural way to the correct behavior of the stabilization parameters while steady-state solutions do not depend on it. Furthermore, in this work we have proved using numerical analysis that dynamic subscales exhibit enhanced stability properties that are independent of the time-space discretization. Tracking the subscales in time even error estimates have been proved without any restriction over the time step size. We refer to Codina et al. [15] for a complete set of numerical experiments that support our analysis.

\section{References}

[1] I. Babuŝka, Error bounds for the finite element method, Numerische Mathematik 16 (1971) 322-333.

[2] S. Badia, R. Codina, Unified stabilized finite element formulations for the Stokes and the Darcy problems, submitted for publication.

[3] J. Blasco, R. Codina, Space and time error estimates for a first order, pressure stabilized finite element method for the incompressible Navier-Stokes equations, Applied Numerical Mathematics 38 (2001) 475-497.

[4] P. Bochev, M. Gunzburger, R. Lehoucq, On stabilized finite element methods for the Stokes problem in the small time-step limit, International Journal for Numerical Methods in Fluids 53 (2007) 573-597.

[5] D. Boffi, L. Gastaldi, Analysis of finite element approximation of evolution problems in mixed form, SIAM Journal on Numerical Analysis 42 (2004) $1502-1526$.

[6] J.M. Boland, R.A. Nicolaides, Stability of finite elements under divergence constraints, SIAM Journal on Numerical Analysis 20 (1983) $722-731$.

[7] S.C. Brenner, L.R. Scott, The Mathematical Theory of Finite Element Methods, Springer-Verlag, 1994.

[8] F. Brezzi, On the existence, uniqueness and approximation of saddle point problems arising from lagrange multipliers, RAIRO Analyse Numerique 8 (1974) 129-151.

[9] F. Brezzi, M. Fortin, Mixed and Hybrid Finite Element Methods, Springer-Verlag, 1991.

[10] E. Burman, M.A. Fernández, Galerkin finite element methods with symmetric pressure stabilization for the transient Stokes equations: stability and convergence analysis, SIAM Journal on Numerical Analysis, in press.

[11] R. Codina, Stabilized finite element approximation of transient incompressible flows using orthogonal subscales, Computer Methods in Applied Mechanics and Engineering 191 (2002) 4295-4321.

[12] R. Codina, Analysis of a stabilized finite element approximation of the Oseen equations using orthogonal subscales, Applied Numerical Analysis 58 (2008) 264-283.

[13] R. Codina, J. Blasco, A finite element formulation for the Stokes problem allowing equal velocity-pressure interpolation, Computer Methods in Applied Mechanics and Engineering 143 (1997) 373-391.

[14] R. Codina, J. Blasco, Analysis of a pressure-stabilized finite element approximation of the stationary Navier-Stokes equations, Numerische Mathematik 87 (2000) 59-81.

[15] R. Codina, J. Principe, O. Guasch, S. Badia, Time dependent subscales in the stabilized finite element approximation of incompressible flow problems, Computer Methods in Applied Mechanics and Engineering 196 (2007) 2413-2430.

[16] R. Codina, O.C. Zienkiewicz, CBS versus GLS stabilization of the incompressible Navier-Stokes equations and the role of the time step as stabilization parameter, Communications in Numerical Methods in Engineering 18 (2002) 99-112.

[17] T. Gelhard, G. Lube, M.A. Olshanskii, J.A. Starcke, Stabilized finite element schemes with lbb-stable elements for incompressible flows, Journal of Computational and Applied Mathematics 177 (2005) 243-267.

[18] P. Hansbo, A. Szepessy, A velocity-pressure streamline diffusion finite element method for the incompressible Navier-Stokes equations, Computer Methods in Applied Mechanics and Engineering 84 (1990) 175-192.

[19] T.J.R. Hughes, Multiscale phenomena: Green's function, the Dirichlet-to-Neumann formulation, subgrid scale models, bubbles and the origins of stabilized formulations, Computer Methods in Applied Mechanics and Engineering 127 (1995) 387-401.

[20] T.J.R. Hughes, G.R. Feijóo, L. Mazzei, J.B. Quincy, The variational multiscale method-a paradigm for computational mechanics, Computer Methods in Applied Mechanics and Engineering 166 (1998) 3-24.

[21] T.J.R. Hughes, L.P. Franca, M. Balestra, A new finite element formulation for computational fluid dynamics: V. Circumventing the Babuŝka-Brezzi condition: a stable Petrov-Galerkin formulation for the Stokes problem accommodating equal-order interpolations, Computer Methods in Applied Mechanics and Engineering 59 (1986) 85-99.

[22] S. Idelsohn, N. Nigro, M. Storti, G. Buscaglia, A Petrov-Galerkin formulation for advection-reaction-diffusion problems, Computer Methods in Applied Mechanics and Engineering 136 (1996) 27-46.

[23] O. Ladyzhenskaya, The Mathematical Theory of Viscous Incompressible Flow, Gordon and Breach, New York, 1969. 
[24] J. Principe, R. Codina, F. Henke, The dissipative structure of variational multiscale methods for incompressible flows, Computer Methods in Applied Mechanics and Engineering, in press, doi:10.1016/j.cma.2008.09.007.

[25] F. Shakib, T.J.R. Hughes, A new finite element formulation for computational fluid dynamics: IX. Fourier analysis of space-time Galerkin/least-squares algorithms, Computer Methods in Applied Mechanics and Engineering 87 (1991) 35-58.

[26] R. Temam, Navier-Stokes equations, North-Holland, 1984. 